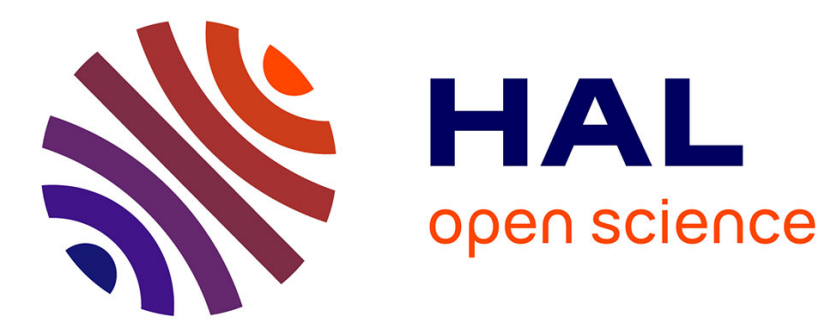

\title{
Photoionization microscopy: Hydrogenic theory in semiparabolic coordinates and comparison with experimental results
}

\author{
P. Kalaitzis, S. Danakas, F. Lepine, C. Bordas, S. Cohen
}

\section{To cite this version:}

P. Kalaitzis, S. Danakas, F. Lepine, C. Bordas, S. Cohen. Photoionization microscopy: Hydrogenic theory in semiparabolic coordinates and comparison with experimental results. Physical Review A, 2018, 97 (5), pp.053412. 10.1103/PhysRevA.97.053412 . hal-02285750

\author{
HAL Id: hal-02285750 \\ https://hal.science/hal-02285750
}

Submitted on 11 Feb 2021

HAL is a multi-disciplinary open access archive for the deposit and dissemination of scientific research documents, whether they are published or not. The documents may come from teaching and research institutions in France or abroad, or from public or private research centers.
L'archive ouverte pluridisciplinaire $\mathbf{H A L}$, est destinée au dépôt et à la diffusion de documents scientifiques de niveau recherche, publiés ou non, émanant des établissements d'enseignement et de recherche français ou étrangers, des laboratoires publics ou privés. 


\title{
Photoionization microscopy: Hydrogenic theory in semiparabolic coordinates and comparison with experimental results
}

\author{
P. Kalaitzis, ${ }^{1}$ S. Danakas, ${ }^{1}$ F. Lépine,${ }^{2}$ C. Bordas,${ }^{2}$ and S. Cohen ${ }^{1, *}$ \\ ${ }^{1}$ Atomic and Molecular Physics Laboratory, Physics Department, University of Ioannina, 45110 Ioannina, Greece \\ ${ }^{2}$ Université de Lyon, CNRS, UMR5306, Institut Lumière Matière, 69622 Villeurbanne, France
}

(Received 14 March 2018; published 25 May 2018)

\begin{abstract}
Photoionization microscopy (PM) is an experimental method allowing for high-resolution measurements of the electron current probability density in the case of photoionization of an atom in an external uniform static electric field. PM is based on high-resolution velocity-map imaging and offers the unique opportunity to observe the quantum oscillatory spatial structure of the outgoing electron flux. We present the basic elements of the quantummechanical theoretical framework of PM for hydrogenic systems near threshold. Our development is based on the computationally more convenient semiparabolic coordinate system. Theoretical results are first subjected to a quantitative comparison with hydrogenic images corresponding to quasibound states and a qualitative comparison with nonresonant images of multielectron atoms. Subsequently, particular attention is paid on the structure of the electron's momentum distribution transversely to the static field (i.e., of the angularly integrated differential cross-section as a function of electron energy and radius of impact on the detector). Such 2D maps provide at a glance a complete picture of the peculiarities of the differential cross-section over the entire near-threshold energy range. Hydrogenic transverse momentum distributions are computed for the cases of the ground and excited initial states and single- and two-photon ionization schemes. Their characteristics of general nature are identified by comparing the hydrogenic distributions among themselves, as well as with a presently recorded experimental distribution concerning the magnesium atom. Finally, specificities attributed to different target atoms, initial states, and excitation scenarios are also discussed, along with directions of further work.
\end{abstract}

DOI: 10.1103/PhysRevA.97.053412

\section{INTRODUCTION}

The quantum description of an atom or a molecule is based on the key concept of the wave function. The wave function itself, however, is traditionally examined only indirectly through a comparison between theoretically calculated and experimentally measured expectation values of some selected observables. Evidently, access to the electronic wave function itself provides more insight into the structure of quantum systems. The strategies followed for reaching this goal include tomographic reconstruction of atomic orbitals in the field of ultrafast physics [1] or weak measurements [2] for reconstructing the complete spatial wave function of a single photon [3]. A common feature of these approaches is that the wave function, or at least the corresponding probability density, is obtained by means of a reconstruction procedure. However, the field of STM or AFM microscopies [4] paved the way toward direct wave function imaging methods. The so-called photoionization microscopy (PM) is one of these very few methods allowing for a direct measurement of the probability density of the electron in the specific context of photoionization in the presence of a static electric field. In this case the wave function of the outgoing photoelectron in the direction of the field extends over macroscopically large distances. Transversely to the field, however, the wave function is bound, leading under appropriate conditions to potentially visible structures in the photoelectron flux. The latter is recorded by a position-sensitive detector and reflects the squared modulus of the electronic wave function.

\footnotetext{
*Corresponding author: scohen@uoi.gr
}

The PM concept of direct wave function imaging was introduced during the early 1980s by Fabrikant [5] and Demkov et al. [6]. It was subsequently analyzed in deeper detail by Kondratovitch and Ostrovsky in a series of papers dealing with the semiclassical description of the hydrogenic Stark effect [7,8]. A first partial experimental realization was achieved in the framework of photodetachment [9], where the wave function observation is limited to purely continuum ones, bearing information on the electron affinity but not on the negative ion target system. On the other hand, neutral atom photoionization allows for the observation of both continuum and quasibound state wave functions. The first PM experiment was performed on xenon atoms [10] and later extended to lithium [11,12], hydrogen [13], and helium [14] atoms.

The above experimental demonstrations were accompanied by the development of theoretical approaches going beyond the semiclassical $[8,15]$ description of the hydrogenic Stark effect. In fact, fully quantum mechanical nonperturbative treatments of the subject were presented in many earlier [16-22] as well as more recent [23-25] works. Present-day PM-oriented theoretical studies [26-29] can be considered as appropriate extensions of these works, while further extensions allowed for their application to multi-electron atomic systems [30-32]. Of course, whenever possible, it is always desirable to obtain insightful analytical results [7,8,25]. Nonetheless, for photoionization studies near the ionization limit (the PM energy range of interest), all-numerical computations appear to be practical, efficient, and more suitable for a proper comparison with experimental data. 
In the present paper we introduce yet another solution of the Coulomb-Stark problem, where Schrödinger equation is separated in semiparabolic coordinates. This coordinate system appears to be quite advantageous for the given problem, easily dealing with the wave function macroscopic extension as well as its peculiarities at small distances. Nevertheless, its employment for quantum calculations in the past was rather limited [19,33], at least as compared to parabolic $[7,8,15,16,18,21,23-25,27,28]$ and mixed $[20,22,26,29]$ coordinates. We propose a numerical implementation based on an array of robust variable-step integration techniques which are simpler and generally more efficient than those employed earlier.

Apart from the purely computational part, however, our main task here is the introduction of the hydrogenic theoretical framework of PM, including the specificities of differential (radial and angular distribution) as well as of total photoionization cross-sections. We particularly focus on the structure of angularly integrated differential cross-sections as a function of electron energy and radius of impact on the detector (in other words, the electron's momentum distribution transversely to the field [27,28]). These 2D maps offer a complete landscape of the relevant quantum interference effects whose gross features remain unnoticed on single images. We present hydrogenic maps corresponding to different initial states and excitation schemes and discuss their general features. Furthermore, excluding specific resonant features of the excitation spectrum, the hydrogenic PM framework can be reliably extended to non-hydrogenic systems. We demonstrate this by a quantitative comparison of computed PM images with resonant experimental data for hydrogen [13] and a qualitative comparison with non-resonant data for nonhydrogenic lithium $[11,12]$ and magnesium atoms. The extension to nonhydrogenic systems is finally established by qualitatively identifying "universal" similarities between an experimental 2D map recorded for magnesium atom and the aforementioned hydrogenic maps. We conclude by discussing the differences that may constitute signatures of non-hydrogenic effects and the directions for further work.

\section{THEORY}

\section{A. Quantum mechanical Coulomb-Stark problem in semiparabolic coordinates}

The Schrödinger equation describing hydrogen atom in the presence of a homogeneous and static electric field $\mathbf{F}$ of strength $F$ and oriented toward the positive $z$ axis is written as (in a.u. $\hbar=e=m_{e}=1$ ),

$$
\left[-\frac{1}{2} \nabla^{2}-\frac{Z}{r}+F z-E\right] \psi(\mathbf{r})=0
$$

with $E$ the energy, $Z$ the nuclear charge (for hydrogen $Z=$ 1 ), and $r=\left[x^{2}+y^{2}+z^{2}\right]^{1 / 2}$. As is well known, Eq. (1) is separable in either parabolic coordinates $\xi=r+z, \eta=r-z$ and $\varphi=\tan ^{-1}(y / x)[7,8,15,16]$, semiparabolic coordinates $[19,33]$,

$$
\begin{aligned}
& \chi=[r+z]^{1 / 2} \geqslant 0, \quad v=[r-z]^{1 / 2} \geqslant 0 \quad \text { and } \\
& \varphi=\tan ^{-1}(y / x),
\end{aligned}
$$

or mixed coordinates $(\chi, \eta, \varphi)[20,22,26,29]$. In the present work we are primarily interested in the $E \geqslant E_{\mathrm{sp}}^{\mathrm{cl}}$ energy range, where the classical saddle point energy is [17]

$$
E_{\mathrm{sp}}^{\mathrm{cl}}=-2[Z F]^{1 / 2} \text { a.u. }
$$

The above range includes the field-free ionization limit, $E=0$, and it is generally characterized by the electron escape to infinity, signaling the need to compute $\psi(\mathbf{r})$ at large (practically macroscopic) distances. To this purpose, it was found computationally more convenient to solve Eq. (1) using semiparabolic coordinates. For example, for a distance of $|z| \sim 10 \mu \mathrm{m}$ from the origin, the parabolic wave function needs to be computed up to $\eta \sim 4 \times 10^{5}$ a.u., while the semiparabolic one up to only $v=\eta^{1 / 2} \sim 6 \times 10^{2}$ a.u.

In semiparabolic coordinates the wave function is written in the form

$$
\psi(\mathbf{r})=[2 \pi \chi v]^{-1 / 2} X(\chi) Y(v) e^{i m \varphi},
$$

with $m=0, \pm 1, \pm 2, \ldots$ the azimuthal quantum number. From Eq. (4) it is evident that wave functions $\psi$ of different $|m|$ are orthogonal to each other. By introducing Eq. (4) into Eq. (1), we obtain the following decoupled differential equations:

$$
\begin{aligned}
& {\left[-\frac{1}{2} \frac{d^{2}}{d \chi^{2}}+\frac{4 m^{2}-1}{8 \chi^{2}}+\frac{F \chi^{4}}{2}-E \chi^{2}-2 Z_{1}\right] X(\chi)=0} \\
& {\left[-\frac{1}{2} \frac{d^{2}}{d v^{2}}+\frac{4 m^{2}-1}{8 v^{2}}-\frac{F v^{4}}{2}-E v^{2}-2 Z_{2}\right] Y(v)=0,}
\end{aligned}
$$

where the separation constants $Z_{1}$ and $Z_{2}$ are related through

$$
Z_{1}+Z_{2}=Z \text {. }
$$

The numerical solution of Eqs. (5a) and (5b) is described in Appendix A. Their structure reveals indeed that the electron is bound along the $\chi$ coordinate, while it can escape to infinity along the $v$ coordinate. The problem as a whole is, therefore, a scattering problem and it is solved for a given, predetermined, energy. Then, for fixed $m, E$, and $F$, Eq. (5a) has the form of a radial Schrödinger equation with an "effective potential,"

$$
U_{X, \mathrm{eff}}(\chi)=\frac{4 m^{2}-1}{8 \chi^{2}}+\frac{F}{2} \chi^{4}-E \chi^{2},
$$

while $2 Z_{1}$ serves as the eigenvalue. An $m=0$ example is given in Fig. 1(a). The small- $\chi$ asymptotic behavior of wave function $X$ is

$$
\underset{\chi \rightarrow 0}{X(\chi)} \rightarrow A_{X} \chi^{|m|+\frac{1}{2}}\left[1+O\left(\chi^{2}\right)\right],
$$

where the $\chi$-normalization constant $A_{X}$ is by definition positive. On the other extreme, the large $\chi$ form of $U_{X \text {,eff }}$ forces $X$ to decay as [18],

$$
\underset{\chi \rightarrow \infty}{X(\chi)} \rightarrow \frac{1}{\chi} \exp \left[-\frac{F^{1 / 2}}{3} \chi^{3}+\frac{E}{F^{1 / 2}} \chi\right] .
$$

The solution of Eq. (5a) leads to the quantization of $Z_{1}$, thus fixing also $Z_{2}$ through Eq. (6). The obtained $Z_{1}^{n_{1},|m|}$ set is characterized by the quantum number $n_{1}=0,1,2 \ldots$, which 

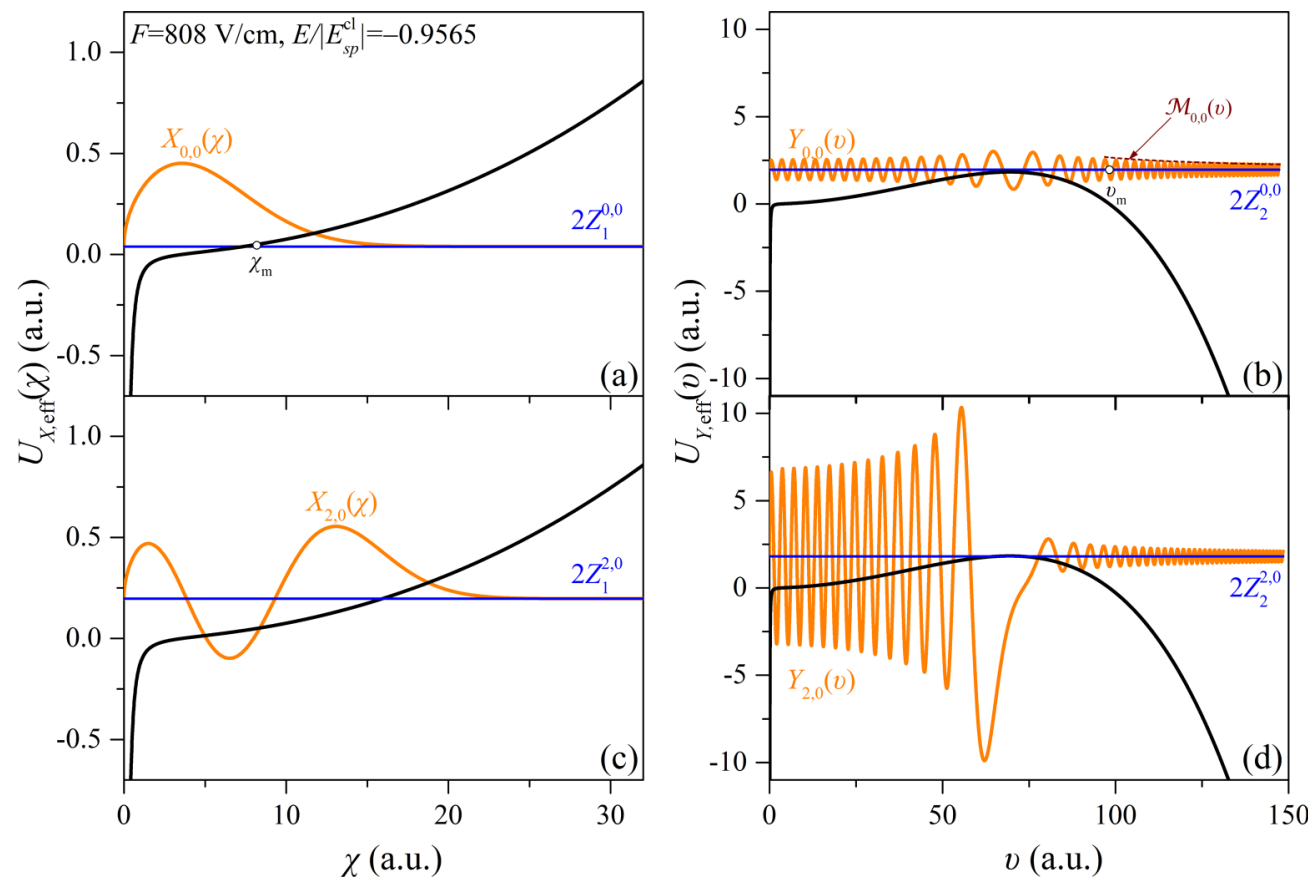

FIG. 1. Semiparabolic "effective potentials" $U_{X \text {,eff }}(\chi)$ and $U_{Y \text {, eff }}(v)$ (black line) along the $\chi$ and $v$ coordinates, respectively, $X_{n_{1},|m|}(\chi), Y_{n_{1},|m|}(v)$ wave functions normalized as described in the text (orange lines) and $2 Z_{1}^{n_{1},|m|}$ and $2 Z_{2}^{n_{1},|m|}$ eigenvalues (horizontal blue lines) for fixed $F=808 \mathrm{~V} / \mathrm{cm}, E /\left|E_{\mathrm{sp}}^{\mathrm{cl}}\right|=-0.9565$ and $m=0$. Note that for $m=0$ the $\left(4 m^{2}-1\right) /\left(4 x^{2}\right), x=\chi, v$ term is a centripetal one. (a, b) The $n_{1}=0$ case for which $2 Z_{2}^{0,0}$ lies above the barrier of $U_{Y, \text { eff }}$ and $Y_{00}$ is a continuum wave function. (c, d) The $n_{1}=2$ case for which $2 Z_{2}^{2,0}$ lies slightly below the barrier and, for this particular energy, $Y_{20}$ corresponds to a quasibound state (resonance, with $n_{2}=27$ ) where the electron is trapped within the inner well. For $n_{1}>2,2 Z_{2}^{n_{1},|m|}$ also lies below the barrier, but these states do not necessarily correspond to resonances and the amplitude of $Y_{n_{1},|m|}$ within the inner well is negligible. The matching points $\chi_{\mathrm{m}}$ and $v_{\mathrm{m}}$ noted in (a) and (b), respectively, which are employed in the computational procedure, are discussed in Appendix A. In (b) the smooth Milne function enveloping $Y_{n_{1},|m|}$ is drawn with brown dashed lines.

is the number of nodes of the corresponding wave functions $X_{n_{1},|m|}$ in the interval $(0, \infty)$. For convenience these wave functions are normalized to unity

$$
\int_{0}^{\infty} X_{n_{1},|m|} X_{n_{1}^{\prime},|m|} d \chi=\delta_{n_{1} n_{1}^{\prime}}
$$

and examples of them are given in Figs. 1(a) and 1(c). For the $v$ coordinate the corresponding "effective potential" writes

$$
U_{Y, \text { eff }}(v)=\frac{4 m^{2}-1}{8 v^{2}}-\frac{F}{2} v^{4}-E v^{2}
$$

[see Fig. 1(b)], and the small- $v$ behavior of wave function $Y$ is

$$
\underset{v \rightarrow 0}{Y} \rightarrow A_{Y} v^{|m|+\frac{1}{2}}\left[1+O\left(v^{2}\right)\right]
$$

$\left(A_{Y} \geqslant 0\right)$. At large distances the large $v$ form of $U_{Y \text {,eff }}$ leads to $[18,26]$

$$
\underset{v \rightarrow \infty}{Y}=C_{Y} \mathscr{M}(v) \sin [\theta(v)+\phi],
$$

where $\mathcal{M}>0$ denotes the Milne function. The latter is obtained from the solution of the Milne equation [34],

$$
\left[\frac{d^{2}}{d v^{2}}+k^{2}\right] \mathcal{M}-\frac{1}{\mathcal{M}^{3}}=0,
$$

into which Eq. (5b) may be transformed [20,26] and

$$
k^{2}(v)=2\left[2 Z_{2}^{n_{1},|m|}-U_{Y, \text { eff }}(v)\right]
$$

is the squared wavenumber function. In fact, at very large distances the $\left(4 m^{2}-1\right) /\left(4 v^{2}\right)$ term in Eq. (11) can be safely neglected and for $v \rightarrow \infty$ we have $\mathcal{M} \approx k^{-1 / 2}$ and the Milne function decreases monotonically and becomes practically independent of both $n_{1}$ and $|m|$. Finally, in Eq. (13),

$$
\theta(v)=\int_{v_{\mathrm{m}}}^{v} \frac{1}{\mathcal{M}^{2}\left(v^{\prime}\right)} d v^{\prime},
$$

and $\phi$ is a constant phase that depends on the lower integration limit $v_{\mathrm{m}}$ in Eq. (16). The wave function $Y$ is energy-normalized and the value of $C_{Y}=[2 / \pi]^{1 / 2}$ is determined by applying standard energy normalization procedures $[16-18,22,26]$. Nevertheless, in accordance with other authors $[17,18]$, we term $A_{Y}$ as the $v$-normalization constant.

The importance of the normalization constants $A_{X, Y}$ can be appreciated by the fact that they provide the probability of finding the electron near the nucleus, i.e., the density of states (DOS). For a given state $\psi_{n_{1}, m}^{E, F}$, we have [22]

$$
\begin{aligned}
\operatorname{DOS}_{n_{1},|m|}(F, E) & \equiv \frac{X_{n_{1},|m|}^{2}(\chi \rightarrow 0}{\chi^{2|m|+1} v^{2|m|+1}}(v \rightarrow 0 \\
& =A_{X, n_{1},|m|}^{2}(F, E) A_{Y, n_{1},|m|}^{2}(F, E),
\end{aligned}
$$

and for a given $|m|$ the total DOS is given by the incoherent sum over all values of $n_{1}$. $A_{X}$ is slowly varying with energy and becomes negligible when $Z_{1}(E)<0$. Similarly, $A_{Y}$ is negligible 
when $Z_{2}(E)<0\left(Z_{1}>Z\right)$. Thus, for a particular $n_{1}$ channel the DOS can be of appreciable magnitude solely within the energy range imposed by the conditions $0 \leqslant Z_{1}^{n_{1},|m|} \leqslant Z$, i.e., the classically allowed range for $Z_{1}$. However, the transition to negligible amplitude for $A_{X}$ and $A_{Y}$ in the neighborhoods $Z_{1} \approx$ 0 and $Z_{1} \approx Z$, respectively, is not that abrupt. It is therefore advisable for any calculation to additionally include at least a few $n_{1}$ terms for which the above conditions are just violated.

Let us finally note that each $n_{1}$ channel is associated with a threshold $E_{\mathrm{thr}}^{n_{1}|m|}$, found by solving the equation $[12,15]$

$$
E=-2\left[Z_{2}^{n_{1},|m|}(E, F) F\right]^{1 / 2} .
$$

For $E>E_{\mathrm{thr}}^{n_{1},|m|}$ [Fig. 1(b)] the electron escapes over the barrier of $U_{Y, \text { eff }}$, and the electron state is a continuum one, characterized only by the pair $\left(n_{1}, m\right)$. However, for $E<$ $E_{\text {thr }}^{n_{1},|m|}$ (i.e., below the $U_{Y \text {,eff }}$ barrier) the electron may escape

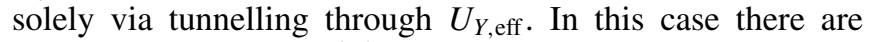
specific values of $Z_{2}^{n_{1},|m|}$, which match the eigenvalues of the inner well of $U_{Y, \text { eff }}$. Then, the wave function $Y$ and consequently $A_{Y}$ acquire large magnitudes within the well [see Fig. 1(d)], reflecting thus the fact that the electron is trapped at short distances and the state exhibits an appreciable lifetime. These so-called quasibound states (resonances) are then characterized by an enlarged set of three quantum numbers $\left(n_{1}, n_{2}, m\right)$, where $n_{2}$ is the number of nodes of $Y$ within the $U_{Y \text {,eff }}$ well. Hence, the structure of DOS as a function of energy is dictated mainly by the energy dependence of $A_{Y}$, which for $E \geqslant E_{\mathrm{sp}}^{\mathrm{cl}}$ and $Z_{1}<Z$ may exhibit zero magnitude, resonant structure due to the presence of resonances, or almost constant nonzero magnitude (continuum). Therefore, the DOS has the appearance of a "spectrum," which is nonetheless independent of any particular initial state or excitation process.

\section{B. Transition matrix elements}

Let us consider the radiative excitation of the final states $\psi_{n_{1}, m}^{E, F}$ out of an initial state $\psi_{i}$ (of energy $E_{i}$ and azimuthal quantum number $m_{i}$ ) via the single-photon dipole transition operator $\hat{T}=\varepsilon \cdot \mathbf{r}$, where $\varepsilon$ is the polarization vector of the exciting laser beam. For calculating the relevant matrix elements,

$$
d_{n_{1}, m}=\left\langle\psi_{n_{1}, m}^{E, F}|\mathcal{E} \cdot \mathbf{r}| \psi_{i}\right\rangle,
$$

we assume that $\psi_{i}$ is either the ground or a low-lying excited state and that for relatively small fields (of the order of a few $\mathrm{kV} / \mathrm{cm}$ ) it is not influenced by its presence. Consequently, the field is neglected for $\psi_{i}$, the latter being a bound unperturbed $(F=0)$ state of free hydrogen. Furthermore, fine-structure effects are not considered here. Since the field-free hydrogenic Hamiltonian is separable in semiparabolic coordinates, $\psi_{i}$ is also written in the form of Eq. (4), which lacks spherical symmetry and has no definite parity. For convenience it will be labeled hereafter as $\left|n, n_{1}, n_{2}, m\right\rangle$, where the principal quantum number $n=n_{1}+n_{2}+|m|+1[16,17]$. The components of $\psi_{i}$ are denoted as $X_{i}$ and $Y_{i}$, respectively. The static field $z$ axis is chosen as the quantization axis. When the linear polarization vector is parallel to the field ( $\pi$ polarization) we have $\varepsilon \cdot \mathbf{r}=z=\left(\chi^{2}-v^{2}\right) / 2$ and the selection rule
$\Delta m \equiv m-m_{i}=0$ applies. Then,

$$
d_{n_{1}, m}^{\pi}=\delta_{m, m_{i}} D_{n_{1},|m|}^{\pi},
$$

where

$$
D_{n_{1},|m|}^{\pi}=\int_{0}^{\infty} d \chi \int_{0}^{\infty} d v X_{i} Y_{i} X_{n_{1},|m|} Y_{n_{1},|m|} \frac{\left(\chi^{4}-v^{4}\right)}{2} .
$$

Despite the oscillatory large- $v$ behavior of $Y_{n_{1},|m|}$ the $v$ integrals appearing in Eq. (21) do not diverge because of the fast decay of the bound wave functions $X_{i}$ and $Y_{i}$ at infinity.

For linear polarization perpendicular to the field ( $\sigma$ polarization) we consider the quite common experimental arrangement of a laser beam propagating perpendicularly to the field (say, along the $x$ axis). Therefore, $\boldsymbol{\varepsilon} \cdot \mathbf{r}=y=\chi v \sin \varphi$, the relevant selection rule is $\Delta m= \pm 1$ and the matrix elements are given by

$$
d_{n_{1}, m}^{\sigma}=\frac{\delta_{m, m_{i}+1}-\delta_{m, m_{i}-1}}{2 i} D_{n_{1},|m|}^{\sigma},
$$

with

$$
D_{n_{1},|m|}^{\sigma}=\int_{0}^{\infty} d \chi \int_{0}^{\infty} d v X_{i} Y_{i} X_{n_{1},|m|} Y_{n_{1},|m|} \chi v\left(\chi^{2}+v^{2}\right) .
$$

In the present work we also consider two-identical-photon excitation of $\psi_{n_{1}, m}^{E, F}$ out of the ground state $\psi_{g}(|1,0,0,0\rangle)$ of energy $E_{g}$ and $m_{g}=0$. Assuming there is no one-photon resonant intermediate state, the two-photon transition matrix elements are, in fact, one-photon matrix elements $d_{n_{1}, m}^{(2)}=$ $\left\langle\psi_{n_{1}, m}^{E, F}|\varepsilon \cdot \mathbf{r}| \psi_{\mathrm{v}}\right\rangle$ connecting $\psi_{n_{1}, m}^{E, F}$ to a virtual state $\psi_{\mathrm{v}}$. The latter may be obtained perturbatively via the Dalgarno-Lewis method [35,36], that is, by solving the inhomogeneous equation,

$$
\left[-\frac{1}{2} \nabla^{2}-\frac{Z}{r}-E_{\mathrm{v}}\right] \psi_{\mathrm{v}}(\mathbf{r})=-\varepsilon \cdot \mathbf{r} \psi_{g}(\mathbf{r}),
$$

with $E_{\mathrm{v}}=\left(E+E_{g}\right) / 2$ the virtual state's energy. The static electric field is again neglected for the ground and virtual states. For solving Eq. (24), the virtual state is written as

$$
\psi_{\mathrm{v}}=(2 \pi \chi v)^{-1 / 2} \sum_{N_{1}, M} \mathrm{X}_{N_{1},|M|}(\chi) \mathrm{Y}_{N_{1}, M}(v) e^{i M \varphi},
$$

where the $M$ sum runs over positive and negative values of $M$ and $N_{1}=0,1,2, \ldots$ The permissible values for the azimuthal quantum number $M$ are determined by selection rules concerning the transition $\psi_{g} \rightarrow \psi_{\mathrm{v}}$, as imposed by the dipole operator in Eq. (24). The solution of Eq. (25) reduces to the calculation of the boundlike wave functions $\mathrm{X}_{N_{1},|M|}$ and $\mathrm{Y}_{N_{1}, M}$ which is described in Appendix B. Once these wave functions have been determined the two-photon matrix elements write

$$
\begin{aligned}
d_{n_{1}, m}^{(2)}= & \sum_{N_{1}, M}\left[\int_{0}^{\infty} d \chi \int_{0}^{\infty} d v \mathrm{X}_{N_{1},|M|} \mathrm{Y}_{N_{1}, M} X_{n_{1},|m|} Y_{n_{1},|m|}\right. \\
& \left.\times\left(\chi^{2}+v^{2}\right) \int_{0}^{2 \pi} \varepsilon \cdot \mathbf{r} \frac{e^{i(M-m) \varphi}}{2 \pi} d \varphi\right]
\end{aligned}
$$


and they are subjected to further selection rules concerning the transition $\psi_{\mathrm{v}} \rightarrow \psi_{n_{1}, m}^{E, F}$.

\section{Electron current probability density and related observables}

Since the electron can escape to infinity solely via the $v$ coordinate, we are interested in the electron flux over the surface of a paraboloid of constant $v \rightarrow \infty$. By projecting the electron current probability density vector $\mathbf{J}$ over the $v$-unit vector $\mathbf{e}_{v}$ we obtain

$$
J_{v} \equiv \mathbf{J} \cdot \mathbf{e}_{v}=\frac{i \pi \alpha \omega}{\left[\chi^{2}+v^{2}\right]^{1 / 2}}\left[\psi_{\text {out }}^{+} \frac{\partial\left(\psi_{\text {out }}^{+}\right)^{*}}{\partial v}-\left(\psi_{\text {out }}^{+}\right)^{*} \frac{\partial \psi_{\text {out }}^{+}}{\partial v}\right],
$$

with $\alpha$ the fine structure constant and $\omega$ the frequency of the photoexciting laser field.

The outgoing wave function $\psi_{\text {out }}^{+}$can be calculated via the time-dependent Schrödinger equation by employing first-order perturbation theory. For a laser field of time-independent amplitude, $\psi_{\text {out }}^{+}$obeys the Dalgarno-Lewis equation [26],

$$
\left[-\frac{1}{2} \nabla^{2}-\frac{Z}{r}+F z-E\right] \psi_{\text {out }}^{+}(\mathbf{r})=-\varepsilon \cdot \mathbf{r} \psi_{i}(\mathbf{r}),
$$

where the initial state $\psi_{i}$ can be either a physical state (singlephoton excitation) or a virtual state (two- or multiphoton excitation). For $\psi_{\text {out }}^{+}$we adopt the form

$$
\psi_{\text {out }}^{+}=(2 \pi \chi v)^{-1 / 2} \sum_{n_{1}, m} X_{n_{1},|m|}(\chi) y_{n_{1}, m}^{+}(v) e^{i m \varphi},
$$

where the wave functions $X_{n_{1},|m|}$ are the solutions of Eq. (5a). In Eq. (29), the $m$ sum runs over positive and negative values of $m$. By plugging Eq. (29) into Eq. (28) and following the derivation described in Ref. [26] (as adapted to semiparabolic coordinates) it turns out that the asymptotic, large- $v$, form of the outgoing wave functions $y_{n_{1}, m}^{+}$can be expressed as

$$
y_{v \rightarrow \infty}^{+}=-\frac{2}{C_{Y}} d_{n_{1}, m} \mathcal{M}_{n_{1},|m|}(v) e^{i\left[\theta_{n_{1},|m|}(v)+\phi_{n_{1},|m|}\right]},
$$

i.e., in terms of the Milne functions $\mathcal{M}_{n_{1},|m|}$, the phases $\left[\theta_{n_{1},|m|}(v)+\phi_{n_{1},|m|}\right]$ and the matrix elements $d_{n_{1}, m}$, all determined by the solution of Eq. (5b). As mentioned above, for $v \rightarrow \infty$ the Milne functions become practically independent of both $n_{1}$ and $|m|$. This does not hold for the phases and matrix elements, carrying information concerning the inner part of the wave function. After appropriate manipulations, the $v \rightarrow \infty$ form of the electron current probability density is written as

$$
\begin{aligned}
\underset{v \rightarrow \infty}{J_{v}=} & \frac{2 \pi \alpha \omega}{\chi v\left[\chi^{2}+v^{2}\right]^{1 / 2}} \\
& \times\left|\sum_{n_{1}, m} e^{i\left[\theta_{n_{1},|m|}(v)+\phi_{n_{1},|m|}\right]} d_{n_{1}, m} e^{i m \varphi} X_{n_{1},|m|}(\chi)\right|^{2}
\end{aligned}
$$

and incorporates the angular distribution of the outgoing electron flux. By integrating Eq. (31) over the whole surface of the $v$-paraboloid $(0 \leqslant \chi<\infty$ and $0 \leqslant \varphi \leqslant 2 \pi)$ we obtain the total electron signal, i.e., the total cross-section $\sigma_{\text {tot }}$.
Indeed, the surface element along the $\chi$ and $\varphi$ coordinates is $d S=\chi v\left[\chi^{2}+v^{2}\right]^{1 / 2} d \chi d \varphi$ and by taking into account the normalization of wave functions $X_{n_{1},|m|}$ [Eq. (10)] and the orthogonality between states of different $m$ we find

$$
\sigma_{\text {tot }}=\int J_{v}(\varphi, \chi) d S=4 \pi^{2} \alpha \omega \sum_{n_{1}, m}\left|d_{n_{1}, m}\right|^{2} .
$$

Let us now assume that an electron detector whose plane is perpendicular to $z$ axis is placed at $z_{\mathrm{det}}=-v_{\mathrm{det}}^{2} / 2$. The radius $\rho$ of electron impacts on the constant $v=v_{\text {det }}$ paraboloid is given by $\rho=\left[x^{2}+y^{2}\right]^{1 / 2}=\chi v_{\text {det }}$. The difference between the detector's plane and this paraboloid results to a variation of $z=z_{\operatorname{det}}\left\{1+\left[\rho /\left(2 z_{\operatorname{det}}\right)\right]^{2}\right\}$. Because of the bound character of wave functions $X_{n_{1},|m|}$ there is a maximum finite value $\chi_{\max }$ and a corresponding radius $\rho_{\max }=\chi_{\max } v_{\mathrm{det}}$, after which they have all practically decayed. For this maximum radius the largest $z$ variation is $\Delta z_{\max }=\left(\chi_{\max } / v_{\mathrm{det}}\right)^{2}$. For $v_{\mathrm{det}} \rightarrow \infty$ this variation is negligible while in practice one has to ensure that $v_{\text {det }} \gg$ $\chi_{\max }$. Then, $\left[\chi^{2}+v_{\mathrm{det}}^{2}\right]^{1 / 2} \approx v_{\mathrm{det}}$ and Eq. (31) is simplified to

$$
J_{v_{\mathrm{det}}}(\varphi, \rho) \propto \frac{1}{\chi}\left|\sum_{n_{1}, m} e^{i\left[\theta_{n_{1},|m|}\left(v_{\mathrm{det}}\right)+\phi_{n_{1},|m|}\right]} d_{n_{1}, m} e^{i m \varphi} X_{n_{1},|m|}(\chi)\right|^{2} .
$$

By angularly integrating Eq. (33) we may obtain the radial distribution,

$$
R(\rho) \propto R(\chi)=\int_{0}^{2 \pi} J_{v_{\mathrm{det}}}(\varphi, \chi) d \varphi
$$

which is of primary interest in the present work. Since $\rho \propto k_{\perp}$, where $k_{\perp}$ denotes the norm of the photoelectron's momentum transversely to the field, $R(\rho)$ can be regarded as the electron's transverse momentum distribution [28]. Note, however, that the term "radial distribution" is more frequently employed for $\rho R(\rho)$, which is proportional to the number of electrons hitting the detector within the $[\rho, \rho+d \rho]$ interval.

Finally, another important quantity that can be extracted from Eq. (33) is the signal at the center of the image $(\chi=0)$. Using Eq. (8) we find that only $m=0$ waves contribute to this so-called glory signal (clarified in more detail below), which is then given by

$$
J_{v_{\text {det }}, \text { Glory }} \propto\left|\sum_{n_{1}} d_{n_{1}, 0} A_{X, n_{1}, 0} e^{i\left[\theta_{n_{1}, 0}\left(v_{\mathrm{det}}\right)+\phi_{n_{1}, 0}\right]}\right|^{2} .
$$

The phases and $A_{X, n_{1}, 0}$ coefficients are characteristic of the final Stark states and independent of the excitation process. However, the glory signal depends strongly on the specifics of the initial state and excitation scheme through the transition matrix elements. Furthermore, one should keep in mind that the glory signal, as well as all other observables presented above, exhibits a pronounced dependence on the electron excitation energy. For convenience, in our discussion below this energy is expressed in terms of the dimensionless reduced energy variable

$$
\varepsilon \equiv \frac{E}{\left|E_{\mathrm{sp}}^{\mathrm{cl}}\right|},
$$


and, when required, it will be explicitly denoted in the relevant observables $\left[J_{v_{\text {det }}}(\varepsilon, \varphi, \rho), R(\varepsilon, \rho), \sigma_{\text {tot }}(\varepsilon)\right.$, etc. $]$.

\section{Classical aspects of electronic transverse momentum distribution}

For facilitating the discussion on the energy evolution of $R(\varepsilon, \rho)$, it would be instructive to briefly remind the reader of its features of classical origin, as emerged from classical simulations $[8,37-40]$ and numerous experimental verifications $[10-13,41]$.

The classical treatment of the Coulomb-Stark problem deals solely with open $n_{1}$-channels, since tunnelling is classically forbidden. A particular characteristic of slow photoelectron imaging is the appearance of two concentric structures and a high intensity central peak in the recorded images. The outer structure stems from classical source-to-detector electron trajectories (hereafter called indirect trajectories), which are complicated, they intersect the negative $z$ axis at least once and appear for $\varepsilon \geqslant-1$. The inner structure appears only for $\varepsilon \geqslant$ $\varepsilon_{\mathrm{dir}} \approx-0.775$ and stems from simple quasi-parabolic (direct) trajectories that do not intersect the $z$ axis. A first classification of the two types of trajectories may be provided via the electron's initial ejection angle $\beta$ with respect to the external electric field, where $\beta=0$ denotes uphill ejection $(+z)$ and $\beta=\pi$ downhill ejection $(-z)$ toward the detector. Let us recall that $\beta$ is related to the separation constants $Z_{1,2}$ (for example, $\left.Z_{1}=Z \cos ^{2}[\beta / 2][37,38]\right)$. For $-1 \leqslant \varepsilon \leqslant 0$ both types of trajectories correspond to launch angles $\beta \geqslant \beta_{c} \equiv \arcsin [|\varepsilon|]$ (for $\beta<\beta_{c}$ the electron does not escape from the atom). There is no such restriction for $\varepsilon \geqslant 0\left(\beta_{c}=0\right)$. The indirect trajectories are distinguished from the direct ones through another critical angle $\beta_{\mathrm{o}}$ for which the corresponding trajectory intersects $z$ axis at infinity. Thus, the indirect trajectories correspond to launch angles within the $\left[\beta_{c}, \beta_{\mathrm{o}}\right]$ interval and the direct ones to the $\left[\beta_{0}, \pi\right]$ one. Note that $\beta_{0}=\pi$ for $\varepsilon=\varepsilon_{\mathrm{dir}}$, while for $\varepsilon<\varepsilon_{\mathrm{dir}} \beta_{\mathrm{o}}$ is meaningless.

From the point of view of classical particle scattering [38], photoionization of the electron is treated as a half collision process whose main features can be found by analyzing the radius of impact on the detector as a function of ejection angle. In other words, $\rho(\beta)$ can be regarded as a generalized scattering deflection function. Restricting ourselves to $m=0$ outgoing electrons, within the $\left[\beta_{c}, \pi\right]$ interval $\rho(\beta)$ exhibits several maxima and several zeros. The latter give rise to glory scattering which is responsible for the high intensity central peak of the images. Moreover, the maxima of $\rho(\beta)$ give rise to rainbow scattering. For $\varepsilon \geqslant \varepsilon_{\text {dir }}$ where direct trajectories exist, there is a single maximum $\rho_{I}$ within the interval $\left[\beta_{0}, \pi\right]$, the so-called primary rainbow radius. All other (secondary bow) maxima $\rho_{I I}, \rho_{I I I}$, etc., occur within the $\left[\beta_{c}, \beta_{\mathrm{o}}\right]$ interval. It additionally holds that $\rho_{I}<\rho_{I I}<\ldots<\rho_{\max }^{\mathrm{cl}}$, where $\rho_{\max }^{\mathrm{cl}}$ is the maximum radius of impact, given by a simple analytical expression $[37,38]$. In practice all secondary maxima are indistinguishable from $\rho_{I I}$. Moreover, $\rho_{I I}$ is somewhat different from $\rho_{\max }^{\mathrm{cl}}$ only at low energy $(\varepsilon \sim-1)$, while all (direct and indirect) bow radii progressively merge together at high positive energy. Note finally that the primary bow is the most intense among all bows and that the glory peak is more intense than any bow.

\section{EXPERIMENTAL SET-UP AND PROCEDURE}

Many of the results of theoretical calculations described above are compared in the next section to experimental data obtained with lithium and magnesium atoms. The lithium experimental set-up has already been described in detail $[11,12,42]$. In the magnesium set-up, $\mathrm{Mg}$ vapor is produced in an electrically heated stainless-steel oven mounted at the top of the laser-atom interaction region. The thermal beam of ground-state $\mathrm{Mg}$ atoms interacts with the frequency-doubled radiation delivered by a pulsed Nd:YAG-pumped dye laser operating at a repetition rate of $10 \mathrm{~Hz}$. The fundamental visible $(610-670 \mathrm{~nm})$ radiation pulses have $\sim 5$-ns duration and a linewidth of $\sim 0.2 \mathrm{~cm}^{-1}$. The frequency-doubled UV radiation is produced by a KDP crystal and its pulse energy amounts to $\sim 1 \mathrm{~mJ}$. It is separated from the visible one by an appropriate filter, its linear polarization may be rotated by a $\lambda / 2$-retarder and it is focused to the vacuum chamber via a $\sim 20$-cm focal length lens. The visible beam is guided toward a wavelength calibration system consisting of a Fabry-Perot interferometer providing relative calibration (free spectral range $0.4729(2) \mathrm{cm}^{-1}$ ) and a discharge lamp offering absolute calibration via the one-photon optogalvanic spectrum of $\mathrm{Ne}$.

In both the $\mathrm{Li}$ and $\mathrm{Mg}$ experiments the atomic and laser beams are both perpendicular to the electron spectrometer (and electric field) axis and the achieved background pressure in the interaction region is in the range $2-7 \times 10^{-7} \mathrm{mbar}$. In the case of Li the final Stark states are single-photon excited out of the $2 s^{2} \mathrm{~S}_{1 / 2}$ lithium ground state (wavelength range 228-232 nm). In the case of Mg, the final Stark states are two-photon excited out of the $3 s^{2}{ }^{1} \mathrm{~S}_{0}$ ground state $(305-335 \mathrm{~nm}$, no near-resonant single-photon intermediate levels). For linear laser polarization along the direction of the static electric field $(\Delta m=0)$ we excite $m=0$ final states. For laser polarization perpendicular to the field $(|\Delta m|=1 /$ per photon $)$ we excite $|m|=1$ final states in $\mathrm{Li}$ and $|m|=0,2$ states in Mg.

The electron spectrometer (microscope) is based on a standard three-electrode velocity-map imaging (VMI) spectrometer design [43]. Photoionization takes place in the center between a solid repeller plate and an extractor plate with a hole in its center. These electrodes are biased at voltages $V_{R}$ and $V_{\mathrm{E}}$, respectively. The following grounded third electrode is identical to the extractor plate. The holes of the last two electrodes create an inhomogeneous electric field allowing the fulfilment of the VMI condition for a given $V_{\mathrm{E}} / V_{\mathrm{R}}$ ratio $[42,43]$. In the vicinity of the limited laser-atom interaction volume, however, the field may be considered as nearly constant within $\pm 0.1 \%$.

Photoionized electrons are accelerated by the field toward the end of a field-free drift tube. An electrostatic magnifying Einzel lens is placed about midway the tube $[42,44]$. The lens consists of three identical and equally-spaced electrodes with holes at their centers. The two outer electrodes are grounded, while the middle one is biased to a voltage $V_{\mathrm{L}}$. The electrons are detected at the end of the drift region by a two-dimensional position-sensitive detector made of a tandem microchannel plate assembly followed by a phosphor screen. A CCD camera records the $2 \mathrm{D}$ distribution of light spots on this screen. Recorded images are transferred to a PC, where they are accumulated over several thousand laser shots. The entire 
spectrometer is shielded by a double $\mu$-metal layer, resulting to a residual magnetic field $<1 \mu \mathrm{T}$ in its interior.

The ratio $V_{\mathrm{E}} / V_{\mathrm{R}}$ ensuring $\mathrm{VMI}$ conditions depends on the spectrometer design and (moderately) on whether the Einzel lens is on or off. Typical electric field values between 500 and $1000 \mathrm{~V} / \mathrm{cm}$ are applied in the interaction region and the lens allows for an up to $\sim 20$-fold magnification of the images. For $\sim 20 \mathrm{meV}$ electrons such a magnification leads to typical image sizes of $\sim 20 \mathrm{~mm}$.

\section{RESULTS AND DISCUSSION}

\section{A. Photoionization microscopy images}

Before proceeding to the discussion of radial distributions it is worth testing the above formulation of the Stark effect through a comparison between its predictions and the experiment. For the resonant phenomena the comparison should inevitably involve hydrogenic data, while nonresonant effects afford a qualitative comparison with nonhydrogenic data.

\section{Hydrogenic resonant and nonresonant $m=0$ images and radial distributions}

Experimental images of hydrogenic Stark resonances were presented in Ref. [13] and were recently reproduced theoretically by employing mixed $(\chi, \eta, \varphi)$ coordinates [29]. We examine here the vicinity of the quasibound $\left(n_{1}, n_{2}, m\right)=(2,27,0)$ Stark state, whose presently calculated $X_{2,0}$ and $Y_{2,0}$ wave functions are given in Fig. 1. This state was experimentally reached via a single-photon transition out of an excited $m=0$ state of hydrogen with principal quantum number $n=2$ and expected to exhibit mixed $s$ and $p$ characters in the presence of the field [13]. Here, the mixed character is taken into account by the semiparabolic form [Eq. (4)] of the two relevant zero-field wave functions, namely $\left|n, n_{1}, n_{2}, m\right\rangle=|2,0,1,0\rangle=2^{-1 / 2}\left[|2 s\rangle+\left|2 p_{m=0}\right\rangle\right]$ and $|2,1,0,0\rangle=2^{-1 / 2}\left[|2 s\rangle-\left|2 p_{m=0}\right\rangle\right]$. Calculations were carried out for $F=808 \mathrm{~V} / \mathrm{cm}$ [13], and $v_{\text {det }}=1000$ a.u. $\left(z_{\mathrm{det}} \approx-25 \mu \mathrm{m}\right)$.

Figure 2 shows the results concerning the $|2,0,1,0\rangle$ initial level. The total excitation cross section $\sigma_{\text {tot }}(\varepsilon)$ is plotted in Fig. 2(a) and consists of a weak background upon which the much more intense $(2,27,0)$ Stark resonance is superimposed. Figure 2(b) shows the resonant $J_{v_{\text {det }}}(\varphi, \chi)$ image, as well as nonresonant images lying below and above it by energy differences equal to the experimental ones (see Fig. 4 in Ref. [13]). All computed images accurately reproduce the experimental data and show no angular dependence (as expected for $m=0$ ). It is evident from Eq. (33) that, even on resonance, $J_{v_{\text {det }}}(\varphi, \chi)$ incorporates contributions from both the $(2,27,0)$ resonance $\left(E<E_{\mathrm{thr}}^{n_{1},|m|}\right)$ and the $\left(n_{1}, m\right)=(0,0)$ and $(1,0)$ continuum channels $\left(E>E_{\mathrm{thr}}^{n_{1}|m|}\right)$. However, due to the much larger excitation strength of the resonance, its characteristics dominate the image. Noteworthy is the abrupt on-resonance change of the number of nodes and image radius increase. The latter is attributed to electron escape via tunneling, which characterizes the quasibound states $[8,26,30]$. The present calculations are also in perfect agreement with the aforementioned earlier theoretical efforts [29] where finestructure effects were also taken into account for the initial

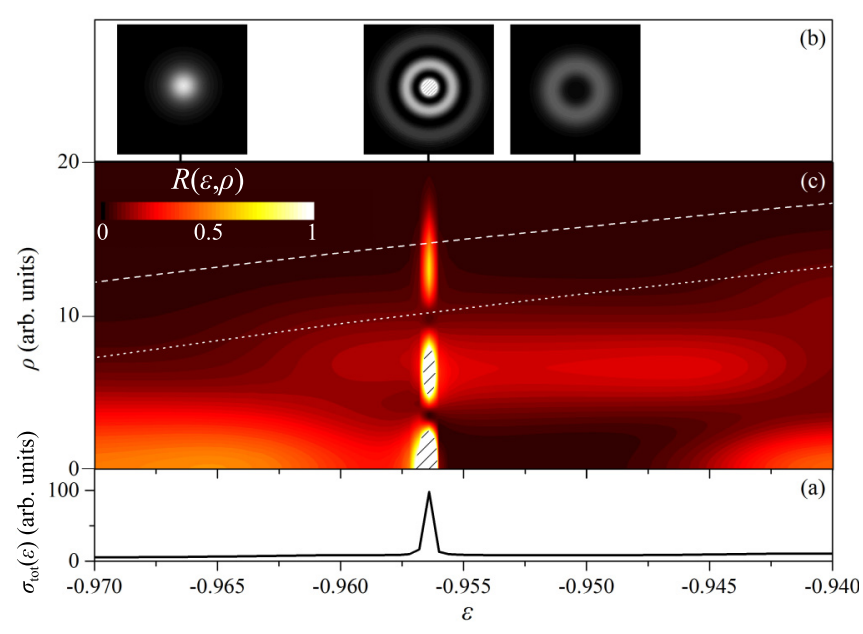

FIG. 2. (a) Hydrogenic total cross section for one-photon excitation out of the $|2,0,1,0\rangle$ initial state to $m=0$ final states in the vicinity of the $\left(n_{1}, n_{2}, m\right)=(2,27,0)$ Stark resonance at $\varepsilon=-0.9564$. The field value is $F=808 \mathrm{~V} / \mathrm{cm}$. (b) Selected $J_{v_{\text {det }}}(\varphi, \chi)$ images, below, on, and above the resonance. The images, computed for $v_{\text {det }}=1000$ $\mathrm{au}$, accurately reproduce the experimental results of Ref. [13] and are in perfect agreement with earlier calculations employing mixed coordinates [29]. Note the abrupt on-resonance fringe number change and image radius increase. (c) Linear scale contour map of the radial distribution $R(\varepsilon, \rho)$ in the vicinity of the resonance. Hatched areas denote intensity cuts. Note the stepwise increase of the nonresonant outer radius at $\varepsilon \approx-0.96$ and $\varepsilon \approx-0.94$, signaling the $n_{1}=1$ and $n_{1}=2$ channel transformations to continua, respectively. Also shown are the maximum classical radius $\rho_{\max }^{\mathrm{cl}}$ (white dashed line) and secondary bow radius $\rho_{I I}$ (white dotted line) [37,38], calculated under the same conditions used for $R(\varepsilon, \rho)$.

state. For simulating the effect of the field, these authors employed an initial state of the form $c_{1}\left|2 s_{1 / 2}\right\rangle+c_{2}\left|2 p_{1 / 2}\right\rangle$ (while $\left|2 p_{3 / 2}\right\rangle$ was ignored) and observed that their results were insensitive to the variation of $c_{1}$ and $c_{2}$. In our case, excitation via the $|2,1,0,0\rangle$ initial level (instead of the $|2,0,1,0\rangle$ one) revealed that, while the resonance still appears in $\sigma_{\text {tot }}(\varepsilon)$, its manifestations on $J_{v_{\text {det }}}(\varphi, \chi)$ almost disappear. In particular, the resonant image hardly shows any fringe number change, while its radius only slightly increases with respect to the nonresonant images of its immediate vicinity. Since the initial state employed in Ref. [29] is a linear combination of both $|2,0,1,0\rangle$ and $|2,1,0,0\rangle$ levels irrespective of the choice of $c_{1}$ and $c_{2}$, this is not an alerting observation. It nevertheless implies that resonant manifestations on the images are highly sensitive to the chosen initial state and excitation scheme.

It is interesting to examine the relevant radial distribution $R(\varepsilon, \rho)$ given in the contour plot of Fig. 2(c). The thresholds of interest are $\varepsilon_{\mathrm{thr}}^{0,0}=E_{\mathrm{thr}}^{0,0} /\left|E_{\mathrm{sp}}^{\mathrm{cl}}\right| \approx-0.99$ (not shown), $\varepsilon_{\mathrm{thr}}^{1,0} \approx$ -0.97 (below the resonance) and $\varepsilon_{\text {thr }}^{2,0} \approx-0.95$ (above it). The plot reveals that for values of $\varepsilon$ just above each threshold the outer radii of nonresonant $R(\varepsilon, \rho)$ distributions initially increase abruptly within a range $\Delta \varepsilon \sim 0.005$ and subsequently remain practically constant. In other words, the range $\varepsilon \sim-1$ is characterized by a stepwise increase of the non-resonant outer radii, in the vicinity of each $\varepsilon_{\mathrm{thr}}^{n_{1},|m|}$ threshold. In contrast, the classical radii $\rho_{I I}$ and $\rho_{\max }^{\mathrm{cl}}[37,38]$, included in Fig. 2(c) and 


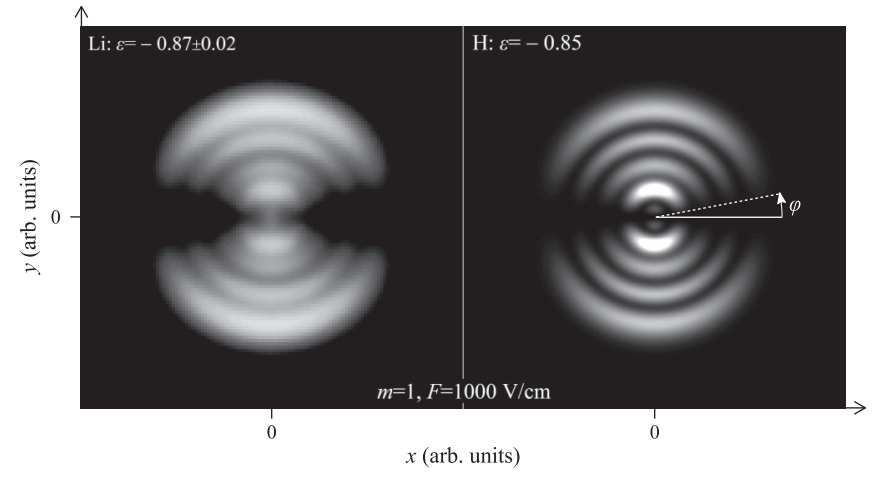

FIG. 3. Comparison between an experimental, nonresonant $m=$ 1 lithium image (left) and a calculated hydrogenic one (right). The horizontal and vertical axes refer to the $x$ and $y$ coordinates, respectively, of electron impact on the detector. $\mathrm{Li}$ atoms were single-photon excited out of the $2 s$ ground state with $\sigma$-polarization (laser beam propagating along $x$ axis and with its linear polarization directed along $y$ axis) and for an estimated field $F=1000 \pm 10$ $\mathrm{V} / \mathrm{cm}$ [12]. For this field and polarization, the hydrogenic image was calculated for single-photon excitation out of the initial $|2,0,1,0\rangle$ state, for $v_{\mathrm{det}}=1000$ au and for a slightly different reduced energy (see text), to reproduce as close as possible the experimental bright fringe intensity distribution. Both images show the expected $\sin ^{2} \varphi$ angular dependence.

calculated under the same conditions, do not exhibit this stepwise increase. Moreover, $\rho_{\max }^{\mathrm{cl}}$ systematically overestimates the nonresonant radii. However, after some experimentation we have verified that $\rho_{I I}$ matches to an excellent approximation the outer turning point of $R(\varepsilon, \rho)$, when the latter is calculated at $\varepsilon=\varepsilon_{\mathrm{thr}}^{n_{1},|m|}+\Delta \varepsilon$. Given that, for the field strengths of interest here, the $\varepsilon_{\text {thr }}^{n_{1},|m|}$ thresholds are expected to be practically independent of the atomic target, this behavior could be useful for a more accurate determination of the field strength in studies devoted to multielectron atoms.

\section{Lithium nonresonant $m=1$ images}

The above discussion suggests that nonresonant $\mathrm{PM}$ images produced by the hydrogenic theory near $\varepsilon \sim-1$ could be compared, at least qualitatively, with those recorded on nonhydrogenic atoms. Our first nonhydrogenic example concerns an $\left(n_{1}, m\right)=(4,1)$ continuum Stark state of lithium atom excited from the $2 s$ ground state in the presence of a field $F=1000 \pm 10 \mathrm{~V} / \mathrm{cm}[11,12]$. To somehow simulate the employed excitation scheme the hydrogenic image was computed assuming $\sigma$-polarization and single-photon ionization out of the $|2,0,1,0\rangle$ hydrogenic state. Figure 3 shows the image resembling the most the experimental one, found by scanning the reduced energy within the $\left[\varepsilon_{\text {thr }}^{4,1}, \varepsilon_{\text {thr }}^{5,1}\right)$ interval. The small difference of reduced energies corresponding to the two images is compatible with the reported field uncertainty. The $\sin ^{2} \varphi$ angular dependence expected for an $|m|=1$ final state is evident. The lower spatial resolution of the experimental image is to be expected since the computed data are not convoluted with the resolution of the detector. Such an operation was avoided because it is unnecessary for a qualitative comparison. The most interesting common feature between the two

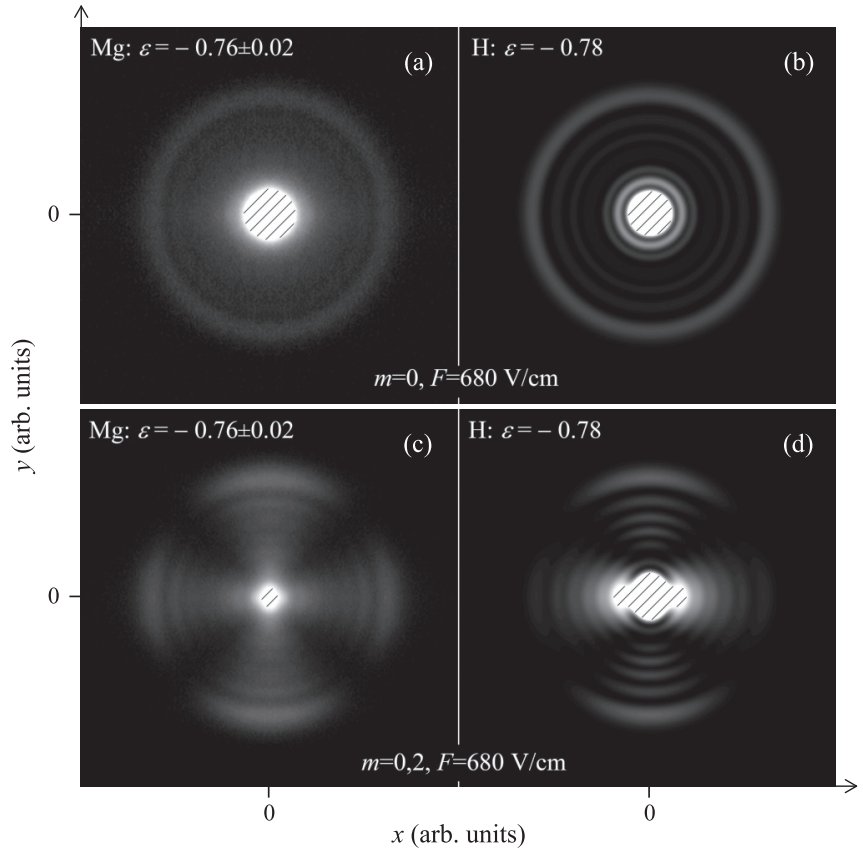

FIG. 4. Comparison between experimental non-resonant magnesium images $(a, c)$ and hydrogenic theoretical images (b, d). The horizontal and vertical axes refer to the $x$ and $y$ coordinates, respectively, of electron impact on the detector. The laser beam propagates along $x$ axis. $\mathrm{Mg}$ final states were two-photon excited out of the $5 s^{2}$ ground state for an estimated static field value $F=680 \pm 10 \mathrm{~V} / \mathrm{cm}$. The hydrogenic calculation refers to two-photon excitation from the $1 \mathrm{~s}$ ground state, for the same field, $v_{\text {det }}=1000$ au and a slightly different reduced energy, to reproduce as close as possible the experimental bright fringe intensity distribution. The light polarization vector is either parallel to the field and $z$ axis $[m=0$ final states $(a, b)]$ or perpendicular to it and directed along $y$ axis [simultaneous $|m|=0$, 2 final state excitation (c, d)].

images is their quite similar bright fringe intensity distribution; particularly the rather unexpected high intensity of the second bright fringe (counting from the image center). By using the $|2,1,0,0\rangle$ initial state (not shown) we observe a quite different bright fringe intensity distribution and this particular fringe has the lowest intensity among all others. This is also a clear indication of the sensitivity of $J_{v_{\mathrm{det}}}(\varphi, \chi)$ on the initial state.

\section{Magnesium nonresonant $m=0$ and $m=0,2$ images}

A second nonresonant example refers to the present measurements of two-photon ionization of ground state magnesium in the presence of a field $F=680 \pm 10 \mathrm{~V} / \mathrm{cm}$. The experimental images were recorded using either $\pi-[m=0$ final Stark states-Fig. 4(a)] or $\sigma$-polarization $[|m|=0$ and 2 final states-Fig. 4(c)]. Experimental data are compared with the theoretical images of Figs. 4(b) and 4(d), computed via two-photon excitation of hydrogen ground state $|1,0,0,0\rangle$. The slightly different reduced energies at which recorded and computed images better resemble to each other are again compatible with our field uncertainty. In fact, despite the different characteristics of the initial states, the $\pi$-polarization images bear many similarities, namely (i) the very intense central glory spot, (ii) a quite intense outer bright (rainbow) 
fringe, and, more interestingly, (iii) the rather faint and low contrast inner bright fringes. Although this last observation may not be generalized, it characterizes the $m=0$ magnesium images recorded by two-photon excitation, as long as the reduced energy is lower than the onset of the direct trajectories $\left(\varepsilon_{\mathrm{dir}} \approx-0.755\right)$.

As for the $\sigma$-polarization images, they also have many features in common. First of all, they both exhibit the expected $\cos ^{2}(2 \varphi)$ angular dependence, which implies the dominance of the $m=2$ waves over the $m=0$ ones. Nevertheless, the $m=0$ contribution manifests itself in a number of ways. First, by the very bright glory signal at the center of each image, whose origin cannot be other than the $m=0$ wave [Eq. (35)]. Second, by an $m$-beating effect [30] [see the coherent summation over different values of $m$ in Eq. (33)], resulting to $\varphi$-dependent bright fringe intensities and radii. The effect is present in both experimental and theoretical images, but more evident in the latter ones. One may notice in Fig. 4(d) that the fringe pattern in the horizontal direction is different and fainter than that in the vertical one. Since, the transition matrix elements may change sign and magnitude as a function of energy, the image direction where the fringes are brighter may also change. Finally, in general, quite complex radial patterns are not uncommon at higher energy.

\section{B. Hydrogenic $R(\varepsilon, \rho)$ maps}

We now proceed to the subject of primary interest in the present work, namely the energy evolution of the radial distributions $R(\varepsilon, \rho)$. To compare with the magnesium experimental map to be discussed in the next subsection, all theoretical maps presented here were computed for a field $F=680 \mathrm{~V} / \mathrm{cm}$ and within the energy range $-1 \leqslant \varepsilon \leqslant+1$. To facilitate the discussion, we employ solely $m=0$ initial and final states ( $\pi$-polarization). The detector is placed at $v_{\operatorname{det}}=1000$ a.u. The graphs of Figs. 5-7 show the total cross sections $\sigma_{\text {tot }}(\varepsilon)$ and maps $R(\varepsilon, \rho)$ for, respectively, single-photon excitation out of the $|1,0,0,0\rangle$ ground state (Fig. 5), single-photon excitation out of the $|2,1,0,0\rangle$ excited state (Fig. 6) and two-photon excitation out of the ground state (Fig. 7). As it is observable in Figs. 5(a)-7(a), for $-1<\varepsilon<0 \sigma_{\text {tot }}(\varepsilon)$ is characterized by a non-resonant background exhibiting an occasional steplike increase and by superimposed resonances of various spectral widths, reflecting their lifetime and tunneling probability. These so-called tunneling states (TS) [24,28] are present in all the spectra with more or less comparable line strengths and profiles. On the contrary, for positive energies $\sigma_{\text {tot }}(\varepsilon)$ is characterized by oscillations which are attributed to so-called Freeman resonances [45] and have been recently termed as static-field-induced-states (SFIS) [24,28]. The contrast of these oscillations depends on the initial state and excitation scheme. For example, it appears to be much larger for single-photon excitation of the $|2,1,0,0\rangle$ state than of the $|2,0,1,0\rangle$ one (this is consistent with experimental observations [46] and earlier calculations [21,22] at fairly higher fields, which have been also successfully reproduced by the present formulation). The data concerning the $|2,0,1,0\rangle$ initial state are not shown here because, though not identical, the corresponding $\sigma_{\text {tot }}(\varepsilon)$ and $R(\varepsilon, \rho)$ observables present many similarities with the two-photon case of Fig. 7. The resemblance is not accidental,

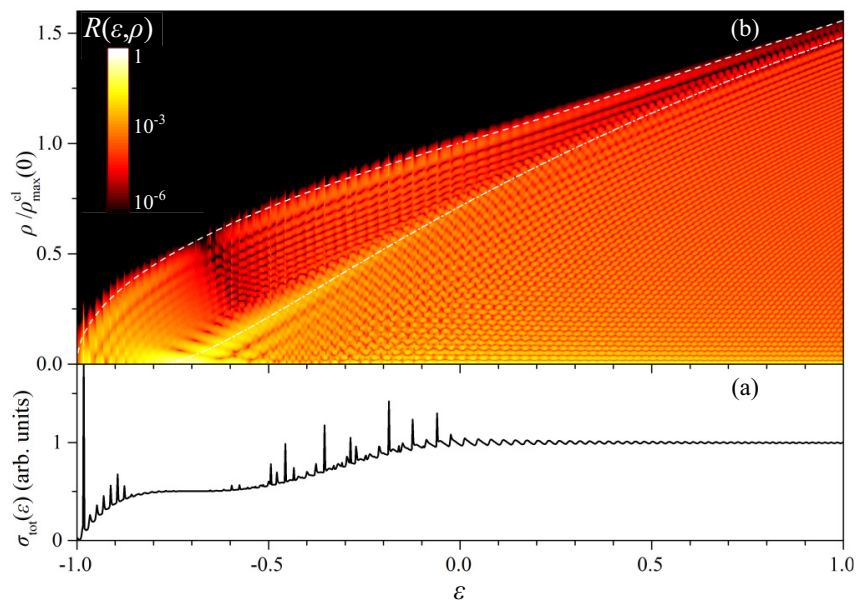

FIG. 5. (a) Hydrogenic total cross section within the $-1 \leqslant \varepsilon \leqslant$ +1 range and $F=680 \mathrm{~V} / \mathrm{cm}$ for single-photon excitation of the $|1,0,0,0\rangle$ ground state to $m=0$ final states. (b) Logarithmic-scale contour map of the radial distribution $R(\varepsilon, \rho)$ computed for the above field and $v_{\text {det }}=1000$ au. The classical maximum radius $\rho_{\max }^{\mathrm{cl}}$ (white dashed line) and primary bow radius $\rho_{I}$ (white dashed-dotted line), computed as described in Refs. [37,38], are also drawn. The map radius $\rho$ is scaled to $\rho_{\max }^{\mathrm{cl}}(\varepsilon=0)$ as described in the text.

since for $\varepsilon / / \mathbf{F}$ the final Stark states are (one-photon) excited from an $N_{1}=M=0$ virtual state, whose $\mathrm{X}$ and $\mathrm{Y}$ wave function components have the same number of nodes with the corresponding $|2,0,1,0\rangle$ state components and bear a close functional form similarity with them.

The radial distributions of TS exhibit much larger radial extensions as compared to continuum Stark states [see Fig. 2(c)]. The most intense of these negative-energy TS resonances are visible in the maps of Figs. 5(b)-7(b). It is not so obvious for the weaker ones, due to the logarithmic false colour magnitude scale of the maps, used to bring out all of their details without any intensity cuts. Apart from these resonances, the general energy evolution of the radial extensions of the maps evidently presents the aforementioned features of classical origin. This is

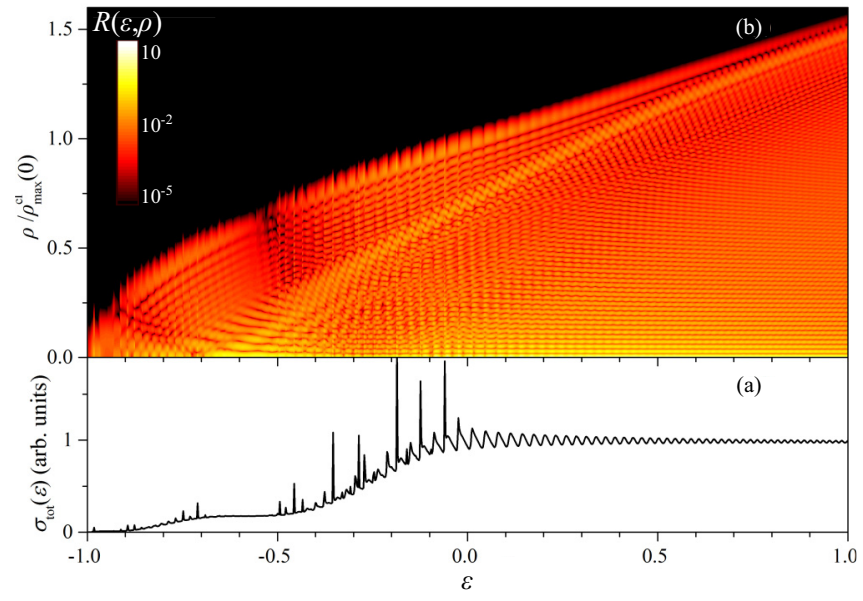

FIG. 6. (a) Hydrogenic total cross-section and (b) logarithmicscale contour map of $R(\varepsilon, \rho)$ for the same field and $v_{\text {det }}$ as in Fig. 5, but for single-photon excitation out of the $|2,0,1,0\rangle$ initial state. 

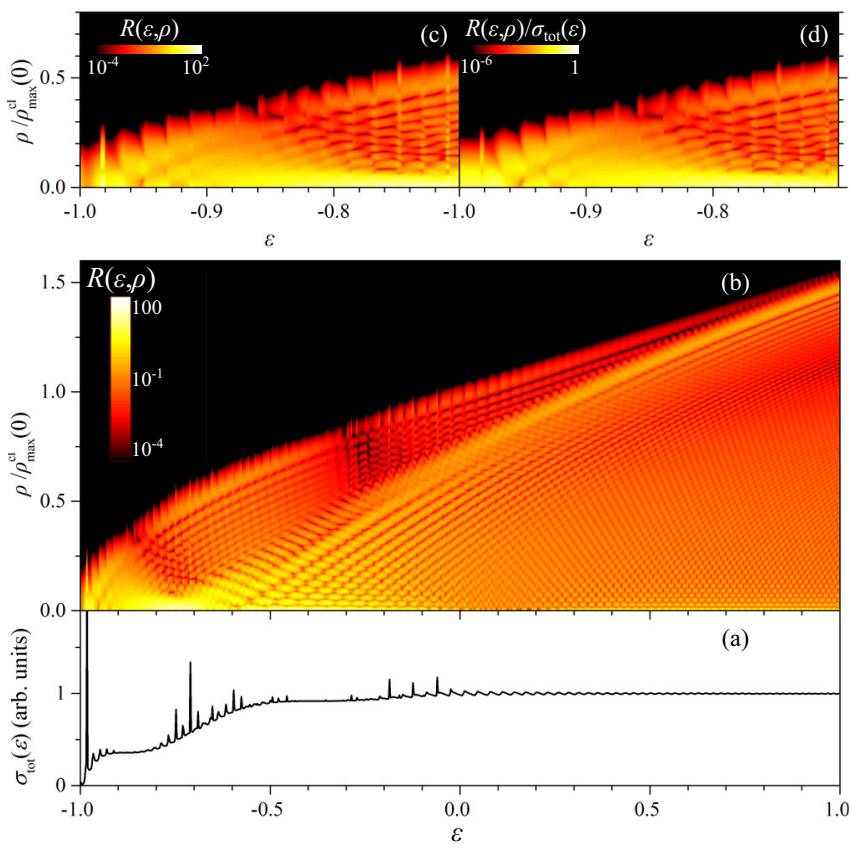

FIG. 7. (a) Hydrogenic total cross-section and (b) logarithmicscale contour map of $R(\varepsilon, \rho)$ for the same field and $v_{\text {det }}$ as in Figs. 5 and 6, but for two-photon excitation out of the $|1,0,0,0\rangle$ ground state. (c) Detail of the map of (b) within the $-1 \leqslant \varepsilon \leqslant-0.7$ range. (d) The scaled radial distribution map $R(\varepsilon, \rho) / \sigma_{\text {tot }}(\varepsilon)$ for the same range of (c). The scaling slightly affects the $n_{1}$-channel opening structures near $\varepsilon \sim-1$ and reduces the strength of resonant manifestations. However, it leaves unaltered the nonresonant fringe patterns over the whole map.

shown in Fig. 5(b) by drawing the maximum radius of impact $\rho_{\max }^{\mathrm{cl}}$ and the primary-bow radius $\rho_{I}$ of the direct contribution $[37,38,40]$. In fact, the radius $\rho$ of all the maps is scaled to $\rho_{\max }^{\mathrm{cl}}(\varepsilon=0)$. At $\varepsilon \approx 0$ this radius is slightly larger than the classical secondary-bow radius $\rho_{I I}(\varepsilon=0)$ by a factor of $\approx 1.01$. However, the quasiperiodic stepwise increase of the nonresonant outer turning points of $R(\varepsilon, \rho)$ discussed earlier [Fig. 2(a)] is seen to persist over the whole $-1 \leqslant \varepsilon \leqslant 1$ range. These oscillating outer turning point radii differ from $\rho_{I I}(\varepsilon \approx 0)$ or $\rho_{\max }^{\mathrm{cl}}(\varepsilon \approx 0)$ by at most $0.5 \%$.

The nature of quantum interferences differs for different parts of the maps. These parts, therefore, deserve a separate discussion. Let us discuss first the zones between $0 \leqslant \rho \leqslant$ $\rho_{\max }^{\mathrm{cl}}$ for $-1 \leqslant \varepsilon \leqslant \varepsilon_{\text {dir }} \approx-0.775$ and $\rho_{I} \leqslant \rho \leqslant \rho_{\max }^{\mathrm{cl}}$ for $\varepsilon \geqslant$ $\varepsilon_{\text {dir }}$, for which the interference pattern is attributed exclusively to the indirect contribution. This pattern is rather simple and, excluding the resonant effects, it basically reflects the nodal structure of the dominant continuum $n_{1}$ channel at a particular energy. This structure is further modulated by the aforementioned channel transformations to continua, which do not cause solely the variation of the outer turning point radii, but they are also responsible for an accompanying quasiperiodic intensity variation of the corresponding radial distributions. The indirect contribution is quite intense at $\varepsilon \sim-1$ and gradually becomes fainter as energy increases. Furthermore, this part of the maps is characterized by slanted quasinodal lines, which create some sort of discontinuity in the behavior of the outer distribution radius as a function of energy. We may notice in Fig. 5(b) that there is a single $\rho>\rho_{I}$ nodal line located within the $-0.8 \leqslant \varepsilon \leqslant-0.6$ range, while in Figs. 6(b) and 7(b) we observe two nodal lines, but not at the same locations. The origin of this indirect-waves-only cancellation effect is at present not fully understood. Nevertheless, since all computed maps deal with the same final Stark states, it is obvious that it should be attributed entirely to the energy evolution of the magnitudes of the relevant excitation matrix elements. We may conclude that the different numbers and locations of nodal lines are a consequence of different energy evolutions of these matrix elements. In turn, these differences originate from the different initial states and excitation schemes. This interpretation explains the absence of these quasinodal lines in semiclassical hydrogenic PM simulations where, as formulated so far [39], excitation matrix elements are not taken into account.

Let us finally turn our attention to the most interesting parts of the maps, $\varepsilon \geqslant \varepsilon_{\text {dir }}$ and $\rho<\rho_{I}$, which exhibit much more complicated quantum interference patterns. The latter reflect the coexistence and subsequent beating effects between direct and indirect contributions, each one characterized by its own fringe "frequencies." Note first the strong quantum oscillations along the classical primary-bow radius $\rho_{I}$, which is common in all maps. Hence, there is no clear boarder between indirect-only and direct-plus-indirect regions, and $\rho_{I}$ serves merely for guiding the eye. Moreover, the gradual transition from the one map range to the other differs in the maps of Figs. 5(b)-7(b). The same holds for the various fringe systems which are formed. Similar structures were observed in recent theoretical calculations [28] and were attributed mainly to the presence of SFIS states. Due, however, to the extreme static fields employed in that work ( $F=0.03-0.1$ a.u. $)$, most of the presently revealed details were usually washed out. Nevertheless, the $\varepsilon>0, \rho=0$ oscillating glory signal [see Eq. (35)] persists also in our weak fields and it is indeed related to the positive energy oscillations of $\sigma_{\text {tot }}(\varepsilon)$ attributed to SFIS. However, although the quasiperiodicity of cross-section oscillations and glory oscillations is practically identical, a closer look reveals that the two signals are dephased and, moreover, that their phase difference varies with energy. This is easily explained by a comparison between Eqs. (32) and (35), which shows that, unlike $\sigma_{\text {tot }}(\varepsilon)$, the glory signal is not simply related to the energy-varying transition matrix elements, but it additionally contains important information on the wave function phase.

Another, most noteworthy, effect of the $\varepsilon>\varepsilon_{\text {dir }}$ and $\rho<$ $\rho_{I}$ map region is the beating pattern observed within the $-0.6 \leqslant \varepsilon \leqslant 0$ range, which resembles a checkerboard. First experimental evidence of the structure was quite recently communicated in Ref. [39] for the hydrogen atom, singly excited from the $|2,0,1,0\rangle$ state under the presence of an $F \approx$ $796 \mathrm{~V} / \mathrm{cm}$ field and within the $-0.45 \leqslant \varepsilon \leqslant-0.2$ range. The checkerboard structure was explained on the basis of classical and semiclassical calculations. It was, moreover, proposed that the structure could be of use for extracting time delays between specific classes of interfering classical trajectories by means of the Eisenbud-Wigner time-delay definition [47]. The semiclassical calculations satisfactorily reproduced the basic characteristics of the checkerboard pattern, buy they were 

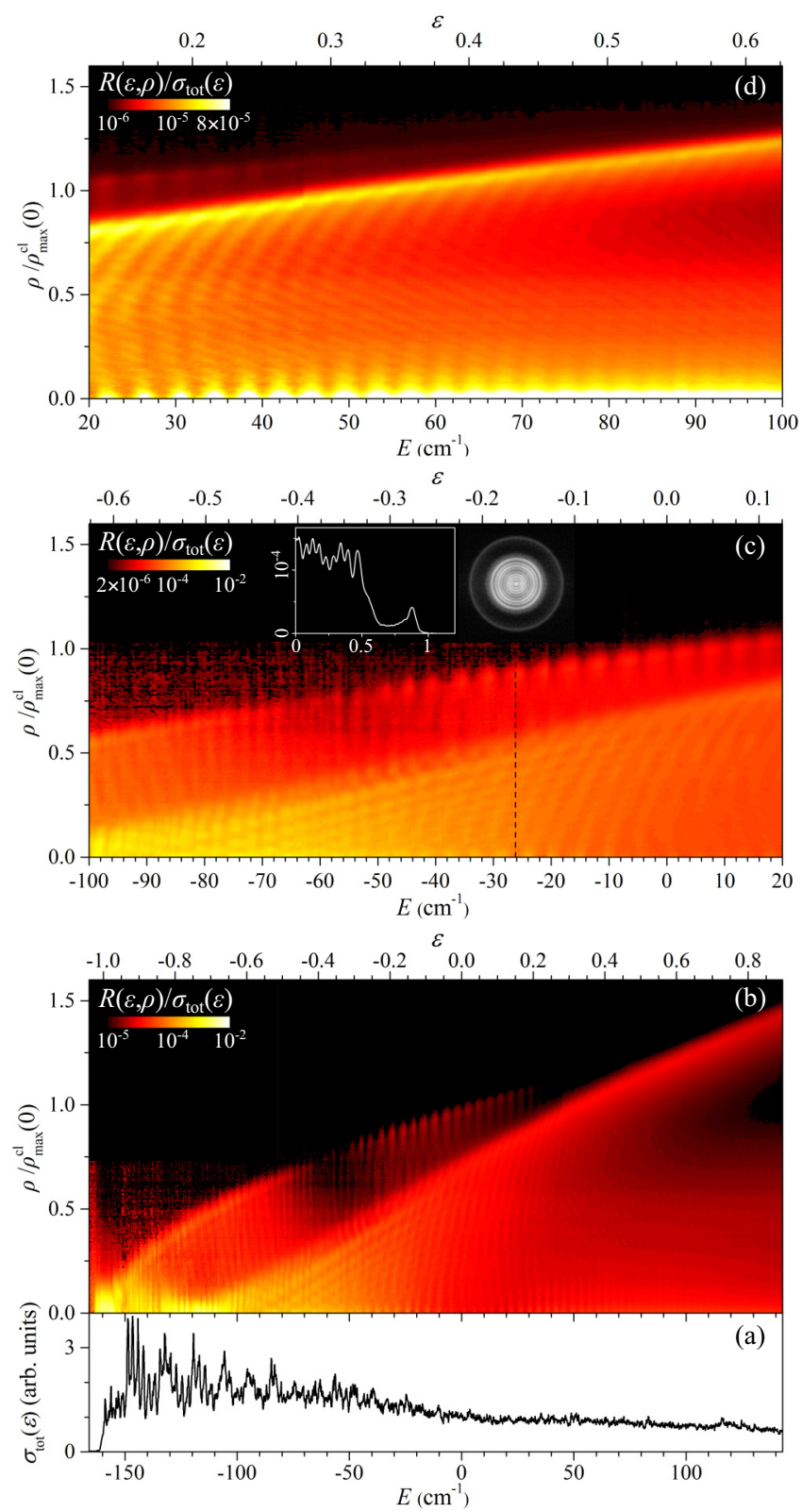

FIG. 8. (a) Experimental total two-photon excitation cross section of $\mathrm{Mg}$ out of its $5 s^{2}$ ground state and under the presence of an $F=$ $680 \pm 10 \mathrm{~V} / \mathrm{cm}$ static field. Laser polarization $\varepsilon / / \mathbf{F}(m=0)$. The scan covers the range $-165 \mathrm{~cm}^{-1} \leqslant E<+145 \mathrm{~cm}^{-1}(-1.03 \leqslant \varepsilon<$ +0.91 , upper $x$ axis). Near $E_{\mathrm{sp}}^{\mathrm{cl}} \approx 159.6 \mathrm{~cm}^{-1}$ we may note the abrupt rise of the $\mathrm{Mg}^{+}$signal and a considerable number of resonances. On the contrary, positive energy Freeman resonances (or SFIS [28]) are not observed, probably because their small modulation depth is comparable to signal's noise. (b) Scaled radial distribution contour map $R(\varepsilon, \rho) / \sigma_{\text {tot }}(\varepsilon)$ over the full energy range $-165 \mathrm{~cm}^{-1} \leqslant E<$ $+145 \mathrm{~cm}^{-1}$. The quasizero $\sigma_{\text {tot }}(\varepsilon)$ values near $\varepsilon \sim-1$ are responsible for the noise observed in that energy range, which is additionally amplified by the color log-scale of the graph. The $y$ axis radius $\rho$ is scaled to $\rho_{\max }^{\mathrm{cl}}$, whose expected behavior (as well as that of $\rho_{I}$ ) is evident. (c) Negative energy detail of (b). The dominant direct-indirect beating feature is the checkerboard structure [39], observed in the range $-0.6 \leqslant \varepsilon<-0.2$. At higher energy this structure evolves into systems of beating fringes. Also noticeable are the indirect-bow magnitude oscillations. The inset shows an image at a given energy (denoted by the black dashed line) and the corresponding radial unable to fully account for the presently revealed complexity of the structure. A drawback of the employed semiclassical approach [10] is that it takes no account of either the particular initial state or the excitation scheme. For example, the $-0.6 \leqslant$ $\varepsilon \leqslant 0$ range of the map of Fig. 5(b) is not characterized by isolated and distinct beating maxima, and, in fact, the observed pattern is not a simple checkerboard structure. The above findings provided the motivation for recordings of experimental maps in the nonhydrogenic magnesium atom. Although it is not our intention here to make an exhaustive analysis of the emerging theoretical and experimental beating patterns, the data presented below may validate or discard our expectations on the emergence of the (nonresonant) complex structures predicted by the hydrogenic theory.

\section{Experimental magnesium $R(\varepsilon, \rho)$ map}

The $m=0$ experimental total cross-section and radialdistribution map recorded in magnesium atom in almost the full $-1 \leqslant \varepsilon \leqslant 1$ range is shown in Figs. 8(a) and 8(b), respectively. Parts of the same map are given in greater detail in Figs. 8(c) and $8(\mathrm{~d})$. The images [an example of which is shown in the inset of Fig. 8(c)] were recorded at a constant energy step of $\Delta E \approx 0.4 \mathrm{~cm}^{-1}\left(\Delta \varepsilon \approx 2 \times 10^{-3}\right)$. The static electric field was estimated via a number of different methods. A first estimate was provided by the $n_{1}=0$ and $n_{1}=1$ channels openings. Next, at positive energies we have compared the observed "frequency" of glory oscillations to the one predicted by hydrogenic theory. Finally, the outermost turning point radius of the high energy $\left(E>-50 \mathrm{~cm}^{-1}\right)$ radial distributions were fitted to the analytical expression for $\rho_{\max }^{\mathrm{cl}}(\varepsilon)[37,38]$. All the methods converged to a field value of $F=680 \pm 10$ $\mathrm{V} / \mathrm{cm}$, which is used for calculating the reduced energy $\varepsilon$, given in the upper $x$ axes of Figs. 8(a)-8(d). By employing the aforementioned 1.01 ratio between the outermost turning point $\left(\rho_{I I}\right)$ and $\rho_{\max }^{\mathrm{cl}}$ at $\varepsilon=0$ we found a refined experimental value for $\rho_{\max }^{\mathrm{cl}}(\varepsilon=0)$ and scaled our radius $\rho$ to it.

Due to the small energy step employed for the measurements, the recording of the map was accomplished within several days. Special care was taken to record unsaturated electron and ion signals, by keeping the laser intensity to the lowest acceptable level (pulse energy $<100 \mu \mathrm{J}$, pulse intensity $<10^{10} \mathrm{~W} / \mathrm{cm}^{2}$ ). However, we were unable to avoid a small gradual decrease of pulse energy with time. For avoiding map magnitude variations attributed to this drift, each experimental radial distribution $R(\varepsilon, \rho)$ is scaled by the total electron signal. Thus, Figs. 8(b)-8(d) do not actually show $R(\varepsilon, \rho)$, but the quantity $R(\varepsilon, \rho) / \sigma_{\text {tot }}(\varepsilon)$. By comparing nonscaled to scaled parts of the maps we have verified that this operation does not affect the details of the interference and beating patterns. This is also true for the calculated hydrogenic maps and an example is given in Figs. 7(c) and 7(d). The scaling slightly diminishes the visibility of the steplike outer radius increase for $\varepsilon \sim-1$

distribution, where beating effects leading to magnitude modulation of the finer fringes are clearly observed. (d) Positive energy detail of (b). The beating effects mentioned in (c) are amplified. Also noteworthy is the strong oscillation of the glory $(\rho=0)$ signal discussed in the text. 
and reduces the importance of tunneling resonances. Yet, the manifestation of such resonances in magnesium is restricted to the vicinity of the saddle point energy.

One may first notice that for the $0 \leqslant \rho \leqslant \rho_{\max }^{\mathrm{cl}},-1 \leqslant \varepsilon \leqslant$ $\varepsilon_{\text {dir }}$, map zone of Fig. 8(b) the faint and low contrast inner bright fringes discussed above for the indirect contribution [see Fig. 4(a)] makes their observation difficult (also due to the logarithmic signal colour scale). However, the channel opening oscillations are quite apparent over the whole indirect contribution. Using scattering terminology [38], we may describe this effect as indirect-bow oscillations. Finally, another characteristic of this section of the map is the quasinodal line (or surface) near $-50 \mathrm{~cm}^{-1}$ and the accompanying discontinuity of $\rho_{\max }^{\mathrm{cl}}$, visible also in the hydrogenic maps.

Passing now to the inner, $\varepsilon>\varepsilon_{\mathrm{dir}}$ and $\rho<\rho_{I}$ region of the map, its most striking feature is the intense checkerboard structure, observed within the $-100 \mathrm{~cm}^{-1} \leqslant E \leqslant$ $-20 \mathrm{~cm}^{-1}(-0.6 \leqslant \varepsilon \leqslant-0.1)$ range. This range is shown in more detail in Fig. 8(c), where the absence of a sharp $\rho_{I}$ boarder discussed in hydrogenic maps is additionally noticeable. As hydrogenic theory and magnesium experimental results imply, if the checkerboard structure is clearly formed, it always appears within the same $\varepsilon$ range. At higher energy it is much less intense and evolves to various beating fringe systems [or lines- see Figs. 8(c) and 8(d)], which are expected to be atom-, initial state-, and excitation scheme-dependent. At $\varepsilon>0$ we may identify in Fig. 8(d) a typical system of slanted fringes, which are also observed in the present theoretical hydrogenic maps and also predicted by the semiclassical theory [39]. Another positive energy feature of the magnesium map that is common with the computed hydrogenic maps concerns the strongly oscillating glory $(\rho=0)$ signal [see Fig. 8(d)]. Certainly these oscillations are related to SFIS resonances [24,28], despite the fact that the resonances themselves are not evident in $\sigma_{\text {tot }}(\varepsilon)$ [Fig. 8(a)], apparently due to their poor contrast. As mentioned above, the "frequency" of the glory signal has been employed for estimating the field strength. To that purpose we made use of the fact that the (dephased) oscillations of the glory signal and the total cross section share the same field-dependent "frequency," and, additionally, the cross-section "frequency" is the same for either hydrogen or nonhydrogenic atoms [15]. Interestingly, however, the magnesium map shows an additional beating pattern that has not been observed in the present theoretical hydrogenic results. This pattern consists of curved, quasivertical beating lines, persisting also to positive energies. The visual effect of these fringes can be noticed in the image and corresponding radial distribution given in the inset of Fig. 8(c). They are responsible for the amplitude modulation of the finer fringes of the image. Such effects were also seen in lithium images [12], but their recording was not as detailed as the present one and they were not discussed in detail. For concluding whether this last fringe pattern is of nonhydrogenic origin, theoretical and further experimental efforts devoted to complex atoms are required.

\section{CONCLUSION}

We have presented a description of photoionization microscopy utilizing the computationally advantageous semiparabolic coordinate system. Theoretically calculated electron probability current density distributions have been successfully compared with experimentally recorded images, resonant for hydrogen atom and nonresonant for more complex atoms. Subsequently, attention has been focused to $m=0$ transverse momentum distributions $R(\varepsilon, \rho)$ of the outgoing electron computed for static electric fields whose strength is small and compatible with current photoionization microscopy studies. Calculations included single- and two-photon excitation out of the ground state of hydrogen, as well as single photon excitation out of an $n=2, m=0$ excited state. The maps revealed quite complex interference and beating patterns whenever the direct and indirect contributions to $R(\varepsilon, \rho)$ overlap. Most noticeable is the emergence of the so-called "checkerboard" pattern, experimentally observed in hydrogen quite recently using a different $n=2, m=0$ excited state. More importantly, this structure appears also in our experimental $R(\varepsilon, \rho)$ map, obtained by two-photon ionization of the ground state of magnesium.

The above analysis has suggested that near the ionization threshold a number of gross features of $R(\varepsilon, \rho)$ are common to all Rydberg atoms in the presence of a static electric field, appear irrespective of the initial state and excitation scheme and may be predicted by fairly simple semiclassical theoretical treatments [39]. That latter work provided a first global classification of direct-indirect beating phenomena. Moreover, recent quantum calculations linked a number of beating fringe systems with positive energy field-induced resonances [28], but many important details of the $R(\varepsilon, \rho)$ maps were washed out by the extreme static field strengths employed in that work. These details have emerged in the present low-field work, revealing a much richer fringe structure, which depends fairly strongly on the initial state, excitation scheme, and as the $\mathrm{Mg}$ experiment has shown, the target atom. These observations call for additional experiments and extended calculations devoted to several multielectron atoms (along with extensions of the present calculations on hydrogen). In fact, it appears that even if $R(\varepsilon, \rho)$ has been recorded for a given target and under given conditions, its different parts (checkerboard structure, glory and bow oscillations, positive energy beating lines, just to name a few) may provide different kind of information and deserve a separate and more detailed analysis than the one provided here. This analysis will be the subject of further work.

\section{ACKNOWLEDGMENTS}

The authors thank CNRS (France) for support through the PICS Program No. 6954. The magnesium experiment was performed at the Central Laser Facility of the University of Ioannina. Technical assistance from P. Triantafillou and E. Dimitriadis is gratefully acknowledged.

\section{APPENDIX A: COMPUTATIONAL DETAILS}

\section{Numerical computation along the $\chi$ coordinate}

The bound wave functions $X_{n_{1}},|m|$ are obtained by the solution of the Schrödinger equation [Eq. (5a)] along the $\chi$ coordinate. This equation is numerically solved for a given set of $|m|, E$, and $F$ values. In order to avoid instabilities occurring at large $\chi$ distances, Eq. (5a) is integrated in both the outward and inward directions for a given value of $Z_{1}$ 
and the logarithmic derivatives of two un-normalized solutions $\left(\tilde{X}^{\prime} / \tilde{X}\right)$ are compared at some matching coordinate $\chi_{\mathrm{m}}$. The latter is slightly larger than the corresponding outer turning point. The value of $Z_{1}$ is modified until the two logarithmic derivatives are equal within a prespecified tolerance. This procedure determines the values of $Z_{1}^{n_{1},|m|}$ and $n_{1}$ [number of zeros of $\tilde{X}$ within the $(0, \infty)$ interval], and, after normalization, the full $X_{n_{1},|m|}$ wave function and the constant $A_{X, n_{1},|m|}$. The outward integration, starts at $\chi_{\mathrm{i}} \sim 10^{-7}$ au and the initial conditions are chosen by making use of the small- $\chi$ asymptotic form of Eq. (7). The inward integration starts at a distance $\chi_{\text {out }}$ ensuring that $\tilde{X}\left(\chi_{\text {out }}\right) / \tilde{X}\left(\chi_{\mathrm{m}}\right) \sim 10^{-10}-10^{-20}[48]$ and the initial conditions are chosen by making use of the $\chi \rightarrow$ $\infty$ asymptotic form of Eq. (8). Due to the complications arising from the fractional power dependence of Eq. (7), most authors proceed to the outward integration of Eq. (5a) by first performing a small- $\chi$ Taylor expansion of $X[18-20,26]$ and by subsequently propagating it via the renormalized Numerov algorithm $[22,26,49]$. Here, we preferred to employ an adaptive stepsize algorithm using the Stoermer's rule as core integrator [50] for solving in both the outward and inward directions. The latter approach is found to be quite robust, reliable, and much simpler, as it does not require the Taylor expansion step.

\section{Numerical computation along the $v$ coordinate}

Along the $v$ coordinate, Eq. (5b) is solved for fixed values of $|m|, E$, and $F$ and $Z_{2}^{n_{1},|m|}=Z-Z_{1}^{n_{1},|m|}$. We proceed again by outward and inward integrations and matching of the two solutions at some intermediate point $v_{\mathrm{m}}$. Outward integration begins again at $v_{\mathrm{i}} \sim 10^{-7}$ a.u. and the initial conditions are chosen by making use of the small- $v$ asymptotic form of Eq. (10). The inward integration starts at $v_{\text {det }} \sim 500-1000$ a.u. These relatively small values correspond to (macroscopic) physical distances of $\sim 10-20 \mu \mathrm{m}$. This fact underlines the advantage of using semi-parabolic coordinates, as compared to the parabolic ones. However, due to the rapid oscillations exhibited by $Y$ at large distances we do not perform the inward integration using Eq. (5b). Instead, we solve the equivalent Milne Eq. (12) [34] and the initial conditions are chosen by making use of $\mathcal{M}\left(v_{\mathrm{det}}\right) \approx k^{-1 / 2}\left(v_{\mathrm{det}}\right)$. These initial values (along with a sufficiently large $v_{\text {det }}$ ) are crucial, in the sense that small departures from them lead to an oscillating Milne function. Furthermore, while the equation is frequently solved with fixed and relatively large stepsizes [20,22], we have found it preferable to use also for its solution the same adaptive stepsize algorithm.

The choice of the matching point $v_{\mathrm{m}}$ is made by consideration of the divergence of the Milne function when it is propagated within classically forbidden regions of space [20]. This concerns the $E<0$ range where the "potential" $U_{Y \text {,eff }}$ along the $v$ coordinate [Eq. (11)] presents a barrier. In this case, $v_{\mathrm{m}}$ is chosen as the point where $U_{\text {eff }}$ crosses $v$-axis after ignoring the diving $\left(4 m^{2}-1\right) /\left(8 v^{2}\right)$ term, i.e., at $v_{\mathrm{m}}=$ $[-2 E / F]^{1 / 2}$. This point is always larger than any turning point; it depends solely on $E$ and $F$ and it corresponds to $Z_{2}=0$, i.e. after (roughly) considering zero attraction of the electron by the nucleus (as in photodetachment). The only drawback of this choice is that $v_{\mathrm{m}}$ becomes very small or even zero near and at $E=0$, respectively. Even more, it cannot be defined for $E>0$ because in this case $U_{\text {eff }}$ is not crossing the $v$ axis at large distances. After some experimentation, the minimum allowed value of $v_{\mathrm{m}}$ is $\left[-2 E_{v} / F\right]^{1 / 2}$ with $E_{v} \approx-0.2 F^{1 / 2}$. This value is also used for $E>0$.

The matching procedure proceeds along the lines described in Ref. [22], as adapted for the semiparabolic coordinates. The procedure provides $Y_{n_{1},|m|}$ over the whole $v$ axis. Particularly, it provides the normalization constant $A_{Y, n_{1},|m|}$ and the phase factor $e^{i\left[\theta\left(v_{\mathrm{det}}\right)+\phi\right]}$ at the detector location $v_{\mathrm{det}}$. The phase $\phi$ depends on $v_{\mathrm{m}}$, but it is not explicitly determined. Finally, having computed the wave functions $X_{n_{1},|m|}$ and $Y_{n_{1},|m|}$, all required integrals and matrix elements with respect to $\chi$ and $v$ are numerically evaluated by applying appropriate adaptive stepsize algorithms [51].

\section{APPENDIX B: COMPUTATION OF VIRTUAL STATES}

Let us now assume two-photon excitation of final Stark states of energy $E$ out of the ground state of energy $E_{g}$ and $m_{g}=0$. The necessary step for calculating two-photon matrix elements is the solution of the inhomogeneous differential Eq. (21), that is $[35,36]$

$$
\left[-\frac{1}{2} \nabla^{2}-\frac{Z}{r}-E_{\mathrm{v}}\right] \psi_{\mathrm{v}}(\mathbf{r})=-\varepsilon \cdot \mathbf{r} \psi_{g}(\mathbf{r}),
$$

with $E_{\mathrm{v}}=\left(E+E_{g}\right) / 2$ the virtual state energy. As mentioned above, the static electric field is neglected for the ground and virtual states, the latter written as

$$
\psi_{\mathrm{v}}=(2 \pi \chi v)^{-1 / 2} \sum_{N_{1}, M} \mathrm{X}_{N_{1},|M|}(\chi) \mathrm{Y}_{N_{1}, M}(v) e^{i M \varphi} .
$$

For the cases of interest here we have $E_{\mathrm{v}}<0$. Plugging Eq. (B2) to Eq. (B1) and after separation of variables we arrive at the following differential equations:

$$
\begin{aligned}
& {\left[-\frac{1}{2} \frac{d^{2}}{d \chi^{2}}+\frac{4 M^{2}-1}{8 \chi^{2}}+\frac{\Omega^{2}}{2} \chi^{2}-2 Z_{1}^{N_{1},|M|}\right] \mathrm{X}_{N_{1},|M|}(\chi)=0} \\
& {\left[-\frac{1}{2} \frac{d^{2}}{d v^{2}}+\frac{4 M^{2}-1}{8 v^{2}}+\frac{\Omega^{2}}{2} v^{2}-2\left(Z-Z_{1}^{N_{1},|M|}\right)\right] \mathrm{Y}_{N_{1}, M}(v)} \\
& =-g_{N_{1}, M}(v),
\end{aligned}
$$

where the functions $g_{N_{1}, M}$ write

$$
\begin{aligned}
g_{N_{1}, M}(v)= & Y_{g}(v) \int_{0}^{\infty} d \chi X_{g} \mathrm{X}_{N_{1},|M|}\left(\chi^{2}+v^{2}\right) \\
& \times \int_{0}^{2 \pi} \mathcal{\varepsilon} \cdot \mathbf{r} \frac{e^{i\left(m_{g}-M\right) \varphi}}{2 \pi} d \varphi .
\end{aligned}
$$

In Eqs. (B3) and (B4) we have defined the "circular frequency,"

$$
\Omega^{2}=2\left|E_{\mathrm{v}}\right|,
$$

while $X_{g}$ and $Y_{g}$ in Eq. (B5) are the $\chi$ and $v$ components of the ground-state wave function $\psi_{g}=|1,0,0,0\rangle$, which are written as

$$
\begin{aligned}
X_{g} & =X_{1,0,0,0}(\chi)=(2 Z)^{1 / 2} \chi^{1 / 2} e^{-\frac{Z}{2} \chi^{2}}, \\
Y_{g} & =Y_{1,0,0,0}(v)=Z v^{1 / 2} e^{-\frac{Z}{2} v^{2}} .
\end{aligned}
$$


Since $m_{g}=0$ and depending on the dipole operator $\varepsilon \cdot \mathbf{r}$, the angular integral in Eq. (B5) allows excitation to virtual states with $|M|=0,1$. Therefore, below we examine solely these two cases. Equation (B3) is a harmonic oscillator Schrödinger equation, whose bound orthonormal solutions are

$$
\mathrm{X}_{N_{1},|M|}(\chi)=A_{N_{1},|M|} \chi^{|M|+1 / 2} e^{-\frac{\Omega}{2} \chi^{2}}{ }_{1} F_{1}\left(-N,|M|+1, \Omega \chi^{2}\right),
$$

where ${ }_{1} F_{1}$ denote confluent hypergeometric functions of the first kind [52], which reduce to Laguerre polynomials $L_{N 1}\left(\Omega \chi^{2}\right)$ for $M=0$. The normalization constants are given by $A_{N_{1}, 0}=[2 \Omega]^{1 / 2}$ and $A_{N_{1}, 1}=\left[2\left(N_{1}+1\right)\right]^{1 / 2} \Omega$ and the corresponding eigenvalues are written as

$$
Z_{1}^{N_{1},|M|}=\Omega\left(N_{1}+\frac{1+|M|}{2}\right) .
$$

It turns out that by imposing $Z_{1}^{N_{1},|M|} \leqslant Z=1$ and for the energy range of interest here $N_{1, \max }=0$ in the sum of Eq. (B2).

Due to the fact that there is no resonant intermediate state at the first photon level Eq. (B4) can be solved by expanding $\mathrm{Y}_{N_{1}, M}$ into the set of eigenfunctions $u_{N_{1}, M}$ of the relevant homogeneous differential equation,

$$
\left[-\frac{1}{2} \frac{d^{2}}{d v^{2}}+\frac{4 M^{2}-1}{8 v^{2}}+\frac{\Omega^{2}}{2} v^{2}-\Lambda_{N_{1}}^{|M|}\right] u_{N_{1}, M}=0,
$$

with $\Lambda_{N_{1}}^{|M|}$ the corresponding eigenvalues. This approach provides analytic forms of $\mathrm{Y}_{N_{1}, M}$, albeit in the form of a series. To this purpose we first set

$$
\mathrm{Y}_{N_{1}, M}=a_{ \pm|M|} \mathrm{y}_{N_{1},|M|},
$$

with the factors $a_{ \pm|M|}$ stemming from the appropriate selection rules. Thus, for $\pi$-polarization $(M=0)$ we have $a_{0}=1$ and for $\sigma$-polarization $(|M|=1) a_{ \pm 1}= \pm 1 / 2 i$. The $N_{1}=0$

functions which are relevant to the examined excitation scheme write

$$
\begin{gathered}
\mathrm{X}_{0,0}(\chi)=(2 \Omega)^{1 / 2} \chi^{1 / 2} e^{-\Omega \frac{\chi^{2}}{2}} \\
\mathrm{y}_{0,0}(v)=2^{4} \Omega^{5 / 2} Z^{3 / 2} v^{1 / 2} e^{-\frac{\Omega}{2} v^{2}}\left[\sum_{\mathrm{n}=0}^{\infty} \frac{\mathrm{n}(Z-\Omega)^{\mathrm{n}-2}[(\mathrm{n}+1) \Omega-2 Z]}{[(\mathrm{n}+1) \Omega-Z](Z+\Omega)^{\mathrm{n}+4}} L_{\mathrm{n}}\left(\Omega v^{2}\right)\right] \\
\mathrm{X}_{0,1}(\chi)=2^{1 / 2} \Omega \chi^{3 / 2} e^{-\frac{\Omega}{2} \chi^{2}} \\
\mathrm{y}_{0,1}(v)=2^{5} \Omega^{3} Z^{3 / 2} v^{3 / 2} e^{-\frac{\Omega}{2} v^{2}}\left[\sum_{\mathrm{n}=0}^{\infty} \frac{(\mathrm{n}+1)(Z-\Omega)^{\mathrm{n}-1}[(\mathrm{n}+2) \Omega-2 Z]}{[(\mathrm{n}+2) \Omega-Z](Z+\Omega)^{\mathrm{n}+5}}{ }_{1} F_{1}\left(-\mathrm{n}, 2, \Omega v^{2}\right)\right] .
\end{gathered}
$$

The infinite sums appearing in Eqs. (B13) and (B15) are in practice restricted to the largely sufficient maximum values $\mathrm{n}_{\max } \approx 15$ for $M=0$ and $\mathrm{n}_{\max } \approx 25$ for $|M|=1$.

[1] J. Itatani, J. Levesque, D. Zeidler, H. Niikura, H. Pépin, J. C. Kieffer, P. B. Corkum, and D. M. Villeneuve, Nature (London) 432, 867 (2004); D. Shafir, Y. Mairesse, D. M. Villeneuve, P. B. Corkum, and N. Dudovich, Nat. Phys. 5, 412 (2009).

[2] Y. Aharonov, D. Z. Albert, and L. Vaidman, Phys. Rev. Lett. 60, 1351 (1988); S. Kocsis, B. Braverman, S. Ravets, M. J. Stevens, R. P. Mirin, L. K. Shalm, and A. M. Steinberg, Science 332, 1170 (2011).

[3] J. S. Lundeen, B. Sutherland, A. Patel, C. Stewart, and C. Bamber, Nature (London) 474, 188 (2011).

[4] J. Repp, G. Meyer, S. M. Stojkovic, A. Gourdon, and C. Joachim, Phys. Rev. Lett. 94, 026803 (2005)

[5] I. I. Fabrikant, Zh. Eksp. Teor. Fiz. 79, 2070 (1980) [JETP 52, 1045 (1980)].

[6] Yu. N. Demkov, V.D. Kondratovich, and V. N. Ostrovsky, Pis'ma Zh. Eksp. Teor. Fiz. 34, 425 (1981) [JETP Lett. 34, 403 (1981)].

[7] V. D. Kondratovich and V. N. Ostrovsky, J. Phys. B 17, 1981 (1984); 17, 2011 (1984); 23, 21 (1990).

[8] V. D. Kondratovich and V. N. Ostrovsky, J. Phys. B 23, 3785 (1990).
[9] C. Blondel, C. Delsart, and F. Dulieu, Phys. Rev. Lett. 77, 3755 (1996).

[10] C. Nicole, H. L. Offerhaus, M. J. J. Vrakking, F. Lépine, and C. Bordas, Phys. Rev. Lett. 88, 133001 (2002); C. Bordas, F. Lépine, C. Nicole, and M. J. J. Vrakking, Phys. Rev. A 68, 012709 (2003); F. Lépine, C. Bordas, C. Nicole, and M. J. J. Vrakking, ibid. 70, 033417 (2004).

[11] S. Cohen, M. M. Harb, A. Ollagnier, F. Robicheaux, M. J. J. Vrakking, T. Barillot, F. Lépine, and C. Bordas, Phys. Rev. Lett. 110, 183001 (2013).

[12] S. Cohen, M. M. Harb, A. Ollagnier, F. Robicheaux, M. J. J. Vrakking, T. Barillot, F. Lépine, and C. Bordas, Phys. Rev. A 94, 013414 (2016).

[13] A. S. Stodolna, A. Rouzée, F. Lépine, S. Cohen, F. Robicheaux, A. Gijsbertsen, J. H. Jungmann, C. Bordas, and M. J. J. Vrakking, Phys. Rev. Lett. 110, 213001 (2013).

[14] A. S. Stodolna, F. Lépine, T. Bergeman, F. Robicheaux, A. Gijsbertsen, J. H. Jungmann, C. Bordas, and M. J. J. Vrakking, Phys. Rev. Lett. 113, 103002 (2014).

[15] D. A. Harmin, Phys. Rev. A 24, 2491 (1981); 26, 2656 (1982). 
[16] H. A. Bethe and E. E. Salpeter, Quantum Mechanics of One and Two-Electron Atoms (Springer, Berlin, 1957), and references therein.

[17] T. F. Gallagher, Rydberg Atoms (Cambridge University Press, Cambridge, 1994), and references therein.

[18] E. Luc-Koenig and A. Bachelier, J. Phys. B 13, 1743 (1980); 13, 1769 (1980).

[19] R. J. Damburg and V. V. Kolosov, J. Phys. B 9, 3149 (1976).

[20] A. Alijah, J. T. Broad and J. Hinze, J. Phys. B 19, 2617 (1986).

[21] G. Alvarez, R. J. Damburg and H. J. Silverstone, Phys. Rev. A 44, 3060 (1991).

[22] A. Alijah, J. Phys. B 25, 5043 (1992).

[23] L. Fernández-Menchero and H. P. Summers, Phys. Rev. A 88, 022509 (2013).

[24] A. V. Gets and O. I. Tolstikhin, Phys. Rev. A 87, 013419 (2013).

[25] V. I. Osherov and V. G. Ushakov, Phys. Rev. A 90, 045401 (2014); 95, 023419 (2017).

[26] L. B. Zhao and J. B. Delos, Phys. Rev. A 81, 053418 (2010).

[27] P. A. Batishchev, O. I. Tolstikhin and T. Morishita, Phys. Rev. A 82, 023416 (2010).

[28] S. Ohgoda, O. I. Tolstikhin and T. Morishita, Phys. Rev. A 95, 043417 (2017).

[29] L. B. Zhao, D. H. Xiao and I. I. Fabrikant, Phys. Rev. A 91, 043405 (2015).

[30] F. Texier, Phys. Rev. A 71, 013403 (2005).

[31] L. B. Zhao, I. I. Fabrikant, J. B. Delos, F. Lépine, S. Cohen and C. Bordas, Phys. Rev. A 85, 053421 (2012); L. B. Zhao, I. I. Fabrikant, M. L. Du and C. Bordas, ibid. 86, 053413 (2012); L. B. Zhao, Europhysics Lett. 109, 23002 (2015).

[32] P. Giannakeas, F. Robicheaux, and C. H. Greene, Phys. Rev. A 91, 043424 (2015).

[33] H. Cartarius, J. Main, T. Losch and G. Wunner, Phys. Rev. A 81, 063414 (2010).

[34] W. E. Milne, Phys. Rev. 35, 863 (1930).

[35] A. Dalgarno and J. T. Lewis, Proc. R. Soc. London, Ser. A 233, 70 (1955).
[36] E. S. Toma and H. G. Muller, J. Phys. B 35, 3435 (2002).

[37] C. Bordas, Phys. Rev. A 58, 400 (1998).

[38] S. Cohen, P. Kalaitzis, S. Danakas, F. Lépine and C. Bordas, J. Phys. B 50, 065002 (2017).

[39] A. S. Stodolna, F. Lépine, A. Rouzée, S. Cohen, A. Gijsbertsen, J. H. Jungmann-Smith, C. Bordas and M. J. J. Vrakking, J. Phys. B 50, 164001 (2017).

[40] T. Barillot, R. Brédy, G. Celep, S. Cohen, I. Compagnon, B. Concina, E. Constant, S. Danakas, P. Kalaitzis, G. Karras, F. Lépine, V. Loriot, A. Marciniak, G. Predelus-Renois, B. Schindler and C. Bordas, J. Chem. Phys. 147, 013929 (2017).

[41] C. Nicole, I. Sluimer, F. Rosca-Pruna, M. Warntjes, M. Vrakking, C. Bordas, F. Texier and F. Robicheaux, Phys. Rev. Lett. 85, 4024 (2000).

[42] M. M. Harb, S. Cohen, E. Papalazarou, F. Lépine and C. Bordas, Rev. Sci. Instrum. 81, 125111 (2010).

[43] A. T. J. B. Eppink and D. H. Parker, Rev. Sci. Instrum. 68, 3477 (1997).

[44] H. L. Offerhaus, C. Nicole, F. Lépine, C. Bordas, F. Rosca-Pruna, and M. J. J. Vrakking, Rev. Sci. Instrum. 72, 3245 (2001).

[45] R. R. Freeman, N. P. Economou, G. C. Bjorklund and K. T. Lu, Phys. Rev. Lett. 41, 1463 (1978).

[46] H. Rottke and K. H. Welge, Phys. Rev. A 33, 301 (1986).

[47] E. P. Wigner, Phys. Rev. 98, 145 (1955).

[48] M. L. Zimmerman, M. G. Littman, M. M. Kash and D. Kleppner, Phys. Rev. A 20, 2251 (1979).

[49] B. R. Johnson, J. Chem. Phys. 67, 4086 (1977).

[50] E. Hairer, S. P. Norsett and G. Wanner, Solving Ordinary Differential Equations I. Nonstiff Problems, 2nd ed., Springer Series in Computational Mathematics (Springer-Verlag, Berlin, 1993).

[51] W. H. Press, S. A. Teukolsky, W. T. Vetterling, B. P. Flannery, Numerical Recipes, 2nd ed. (Cambridge University Press, Cambridge, 1992).

[52] M. Abramowitz and I. A. Stegun, Handbook of Mathematical Functions with Formulas, Graphs, and Mathematical Tables (Dover, New York, 1972). 